Prepared in cooperation with the San Antonio Water System

\title{
Geochemical and Hydrologic Data for San Marcos Springs Recharge Characterization near San Marcos, Texas, November 2008-December 2010
}

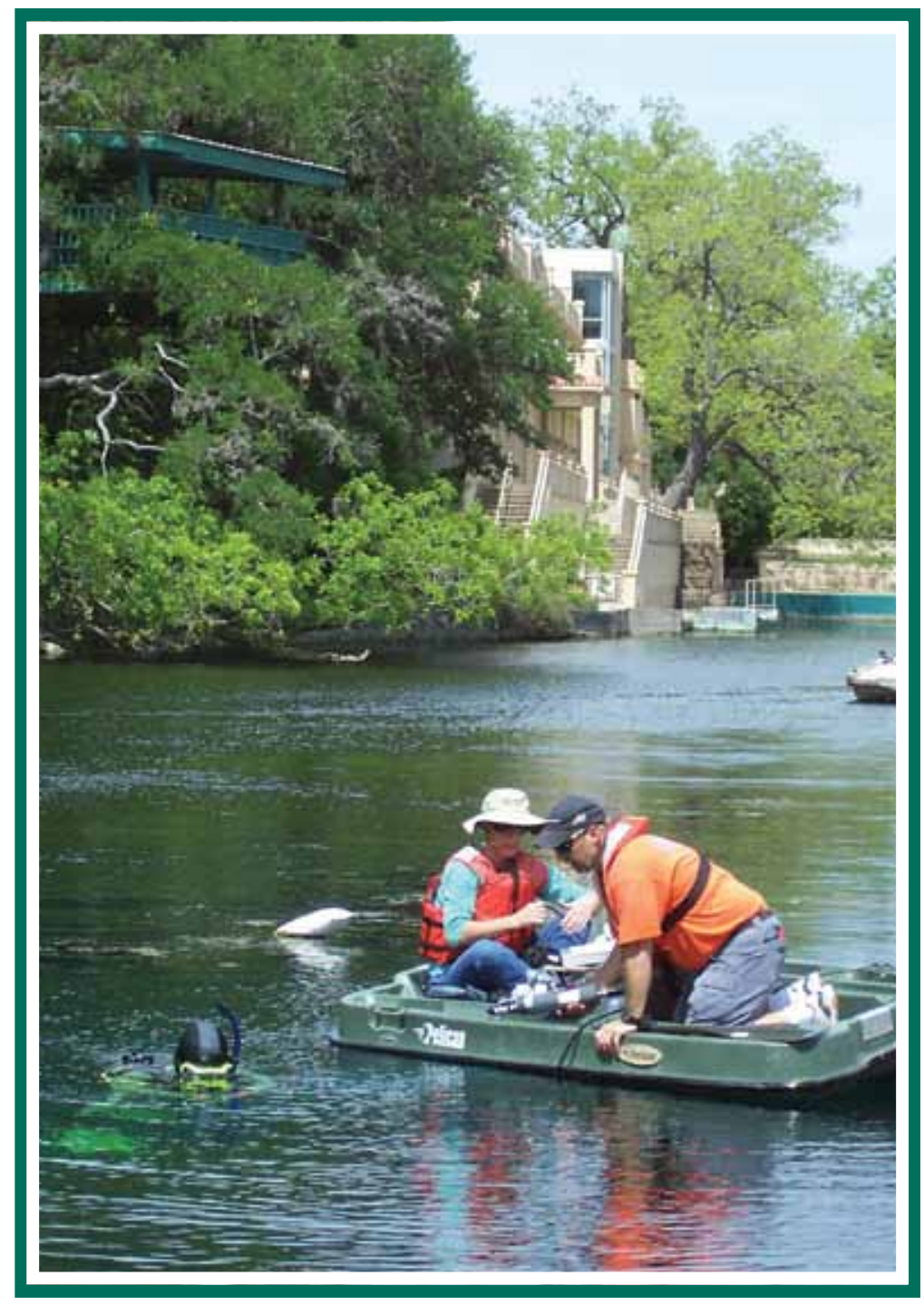

Data Series 672 
Cover, U.S. Geological Survey personnel and a diver from Texas State University install a water-quality monitor at Diversion Spring in the San Marcos Springs complex, Hays County, Texas. Photograph by Cassi Crow, U.S. Geological Survey. 


\section{Geochemical and Hydrologic Data for San Marcos Springs Recharge Characterization near San Marcos, Texas, November 2008- December 2010}

By Cassi L. Crow

Prepared in cooperation with the San Antonio Water System

Data Series 672 


\title{
U.S. Department of the Interior \\ KEN SALAZAR, Secretary \\ U.S. Geological Survey \\ Marcia K. McNutt, Director
}

\section{U.S. Geological Survey, Reston, Virginia: 2012}

\author{
This and other USGS information products are available at http://store.usgs.gov/ \\ U.S. Geological Survey \\ Box 25286, Denver Federal Center \\ Denver, CO 80225 \\ To learn about the USGS and its information products visit http://www.usgs.gov/ \\ 1-888-ASK-USGS
}

\begin{abstract}
Any use of trade, product, or firm names is for descriptive purposes only and does not imply endorsement by the U.S. Government.

Although this report is in the public domain, permission must be secured from the individual copyright owners to reproduce any copyrighted materials contained within this report.
\end{abstract}

Suggested citation:

Crow, C.L., 2012, Geochemical and hydrologic data for San Marcos Springs recharge characterization near San Marcos, Texas, November 2008-December 2010: U.S. Geological Survey Data Series 672, 19 p. (appendixes available online only). 


\section{Contents}

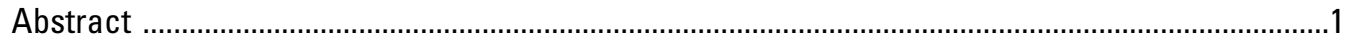

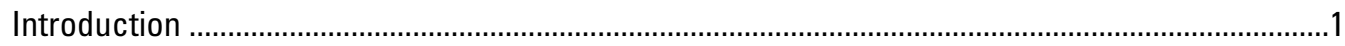

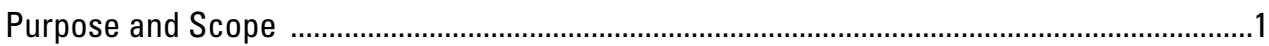

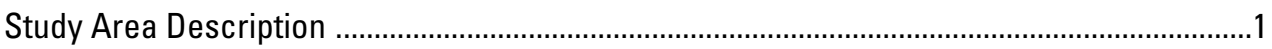

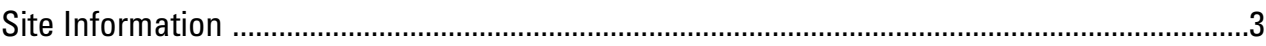

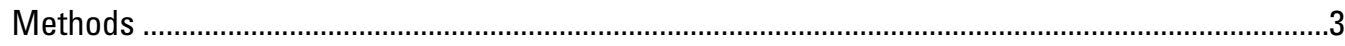

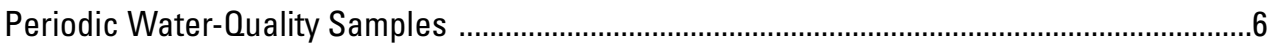

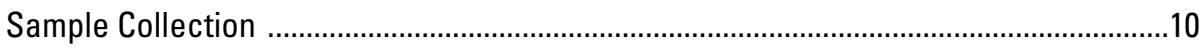

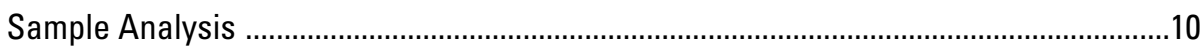

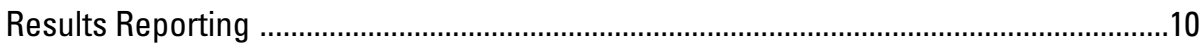

Quality Control .........................................................................................................

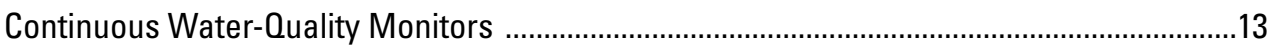

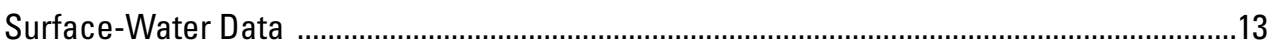

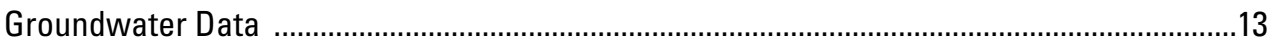

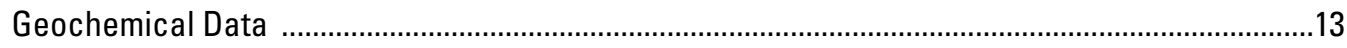

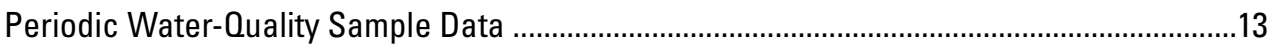

Total Dissolved Solids and Major lons ........................................................................

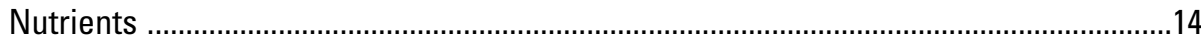

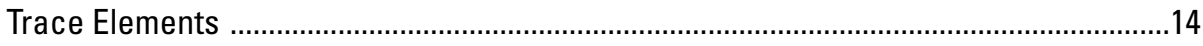

Dissolved Organic Carbon and Total Organic Carbon ...................................................14

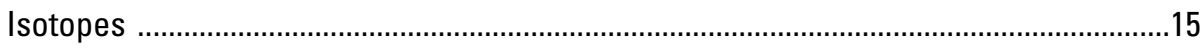

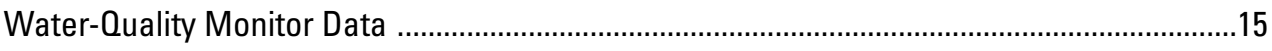

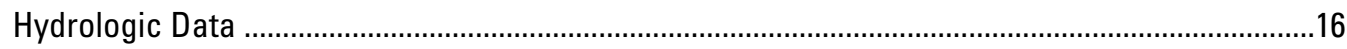

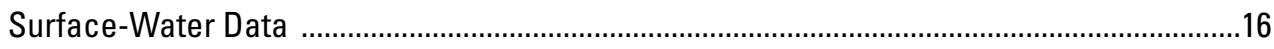

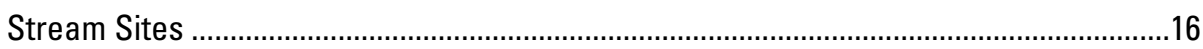

Periodic Discharge Measurements .......................................................................16

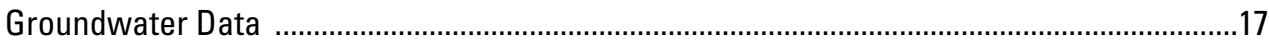

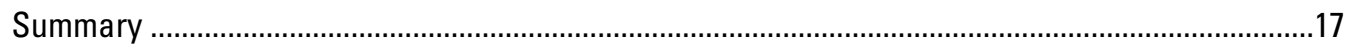

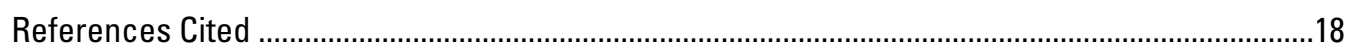

Appendixes (available online only)

1. Water-quality and isotope data collected for San Marcos Springs recharge study, Bexar, Comal, and Hays Counties, Texas, November 2008-December 2010

2. Blank samples collected for San Marcos Spring recharge study, Bexar, Comal, and Hays Counties, Texas, November 2008-December 2010

3. Physicochemical data collected at U.S. Geological Survey station 08171290 Blanco River at Halifax Ranch near Kyle, Texas, water years 2009-11

4. Physicochemical data collected at U.S. Geological Survey station 295314097565701 LR-67-01-826 (TSU-West Campus well), Hays County, Texas, 2009-11

5. Physicochemical data collected at U.S. Geological Survey station 295443097554201 LR-67-01-809 (Tipps well), Hays County, Texas, 2009-10

6. Physicochemical data collected at U.S. Geological Survey station 295322097561000 LR-67-01-819 (Deep Spring), Hays County, Texas, 2009-11

7. Physicochemical data collected at U.S. Geological Survey station 295336097555201 LR-67-01-825 (Diversion Spring), Hays County, Texas, 2009-11 
8. Physicochemical data collected at U.S. Geological Survey station 295322097561002 LR-67-01-820 (Weissmuller Spring), Hays County, Texas, 2010-11

9. Daily mean gage height, in feet, at U.S. Geological Survey station 08169932 Sink Creek near San Marcos, Texas, 2009-11

10. Daily mean gage height, in feet, at U.S. Geological Survey station 08169958 Purgatory Creek at Mountain High Drive near San Marcos, Texas, 2009-11

11. Daily mean gage height, in feet, at U.S. Geological Survey station 08171290 Blanco River at Halifax Ranch near Kyle, Texas, 2009-11

12. Daily mean discharge, in cubic feet per second, computed for U.S. Geological Survey station 08169932 Sink Creek near San Marcos, Texas, 2009-11

13. Daily mean discharge, in cubic feet per second, computed for U.S. Geological Survey station 08171290 Blanco River at Halifax Ranch near Kyle, Texas, 2009-11

14. Instantaneous discharge measurements, in cubic feet per second, from U.S. Geological Survey stations 08169932 Sink Creek near San Marcos, Texas, and 08171290 Blanco River at Halifax Ranch near Kyle, Texas, 2009-11

15. Daily mean depth to water below land surface, in feet, at U.S. Geological Survey station 295314097565701 LR-67-01-826 (TSU-West Campus well), Hays County, Texas, 2009-11

16. Daily mean depth to water below land surface, in feet, at U.S. Geological Survey station 295443097554201 LR-67-01-809 (Tipps well), Hays County, Texas, 2009-10

\section{Figures}

1. Map showing study area for San Marcos Springs recharge study, Bexar, Comal, and Hays Counties, Texas.

2. Map showing locations of spring, stream, groundwater, and precipitation sites for San Marcos Springs recharge study, Bexar, Comal, and Hays Counties, Texas . .4

3. Aerial photographs showing San Marcos Springs complex, Hays County, Texas 5

\section{Tables}

1. Water restriction stage-threshold conditions at the three indicator sites for the Edwards aquifer, south-central Texas

2. Surface-water sites from which data were collected for the San Marcos Springs recharge study, Bexar, Comal, and Hays Counties, Texas

3. Wells and springs from which data were collected for the San Marcos Springs recharge study, Comal and Hays Counties, Texas

4. Sampling cycles and number of samples collected during each cycle (environmental and quality assurance), San Marcos Springs recharge study, Bexar, Comal, and Hays Counties, Texas

5. Summary of long-term method detection levels and laboratory reporting levels for constituents sampled for San Marcos Springs recharge study, Bexar, Comal, and Hays Counties, Texas, November 2008-December 2010

6. Summary of constituent detections greater than or equal to the laboratory reporting level in samples collected for total dissolved solids and major-ion analysis for San Marcos Springs recharge study, Bexar, Comal, and Hays Counties, Texas, November 2008-December 2010 
7. Summary of constituent detections greater than or equal to the laboratory reporting level in samples collected for nutrient analysis for San Marcos Springs recharge study, Bexar, Comal, and Hays Counties, Texas, November 2008December 2010

8. Summary of constituent detections greater than or equal to the laboratory reporting level in samples collected for trace element analysis for San Marcos Springs recharge study, Bexar, Comal, and Hays Counties, Texas, November 2008December 2010

9. Summary of constituent detections greater than or equal to the laboratory reporting level in samples collected for dissolved organic carbon and total organic carbon analysis for San Marcos Springs recharge study, Bexar, Comal, and Hays Counties, Texas, November 2008-December 2010

10. Summary of constituent detections in samples collected for isotope analysis for San Marcos Springs recharge study, Bexar, Comal, and Hays Counties, Texas, November 2008-December 2010

\section{Conversion Factors, Datum, Water-Quality Units, and Isotope Unit Explanations}

\section{Inch/Pound to SI}

\begin{tabular}{lcl}
\hline \multicolumn{1}{c}{ Multiply } & By & \multicolumn{1}{c}{ To obtain } \\
\hline inch (in.) & Length & \\
foot (ft) & 25.4 & millimeter $(\mathrm{mm})$ \\
mile (mi) & 0.3048 & meter $(\mathrm{m})$ \\
\hline & 1.609 & kilometer $(\mathrm{km})$ \\
\hline acre & Area & \\
square mile $\left(\mathrm{mi}^{2}\right)$ & 0.4047 & hectare $(\mathrm{ha})$ \\
\hline & 2.590 & square kilometer $\left(\mathrm{km}^{2}\right)$ \\
\hline gallon per minute $(\mathrm{gal} / \mathrm{min})$ & Flow rate & liter per second $(\mathrm{L} / \mathrm{s})$ \\
million gallons per day $(\mathrm{Mgal} / \mathrm{d})$ & 0.06309 & cubic meter per $\mathrm{second}\left(\mathrm{m}^{3} / \mathrm{s}\right)$ \\
\hline & 0.04381 & \\
\hline foot squared per day $(\mathrm{ft} / \mathrm{d})$ & Transmissivity & meter squared $\mathrm{per} \mathrm{day}\left(\mathrm{m}^{2} / \mathrm{d}\right)$ \\
\hline
\end{tabular}

Temperature in degrees Celsius $\left({ }^{\circ} \mathrm{C}\right)$ may be converted to degrees Fahrenheit $\left({ }^{\circ} \mathrm{F}\right)$ as follows:

$$
{ }^{\circ} \mathrm{F}=\left(1.8 x^{\circ} \mathrm{C}\right)+32
$$

\section{Datums}

Horizontal coordinate information is referenced to the North American Datum of 1983 (NAD 83). 


\section{Water-Quality Units}

Concentrations are reported in metric units. Chemical concentrations are reported in milligrams per liter $(\mathrm{mg} / \mathrm{L})$, micrograms per liter $(\mu \mathrm{g} / \mathrm{L})$, and milliequivalents per liter (meq/L). Milligrams per liter and micrograms per liter are units expressing the concentration of chemical constituents in solution as weight of solute (milligrams or micrograms) per unit volume (liter) of water. For concentrations less than $7,000 \mathrm{mg} / \mathrm{L}$, the numerical value of milligrams per liter is equivalent to the concentration in parts per million. The numerical value of micrograms per liter is equivalent to the concentration in parts per billion. Milliequivalents per liter is a unit expressing the concentration of chemical constituents in solution as $1 / 1,000$ the molecular weight, in milligrams, divided by the valence of one molecule of the constituent per unit volume (liter) of water.

Specific conductance is reported in microsiemens per centimeter at 25 degrees Celsius $(\mu \mathrm{S} / \mathrm{cm})$.

Turbidity is reported in formazin nephelometric units (NFU).

\section{Isotope Unit Explanation}

Per mil: A unit expressing the ratio of stable-isotope abundances of an element in a sample to those of a standard material. Per mil units are equivalent to parts per thousand. Stable-isotope ratios are computed as follows (Coplen and others, 2002):

$$
\delta \mathrm{X}=\left(\mathrm{R}_{\text {sample }} / \mathrm{R}_{\text {standard }}-1\right) \times 1,000,
$$

where

$\delta$ is the "del" notation,

$\mathrm{X}$ is the heavier stable isotope, and

$R$ is the ratio of the heavier, less abundant isotope to the lighter, stable isotope in a sample or standard.

The $\delta$ values for stable-isotope ratios discussed in this report are referenced to the following standard materials:

\begin{tabular}{lll}
\hline \multicolumn{1}{c}{ Element } & \multicolumn{1}{c}{$\mathbf{R}$} & \multicolumn{1}{c}{ Standard identity and reference } \\
\hline carbon & carbon-13/carbon-12 & Vienna PeeDee Belemnite (Fritz and Fontes, 1980) \\
hydrogen & hydrogen-2/hydrogen-1 & Vienna-Standard Mean Ocean Water (Fritz and Fontes, 1980) \\
oxygen & oxygen-18/oxygen-16 & Vienna-Standard Mean Ocean Water (Fritz and Fontes, 1980) \\
\hline
\end{tabular}




\title{
Geochemical and Hydrologic Data for San Marcos Springs Recharge Characterization near San Marcos, Texas, November 2008-December 2010
}

\author{
By Cassi L. Crow
}

\begin{abstract}
During 2008-10, the U.S. Geological Survey, in cooperation with the San Antonio Water System, collected geochemical and hydrologic data in Bexar, Comal, and Hays Counties, Texas, to define and characterize the sources of recharge to San Marcos Springs. Precipitation samples were collected for stable isotope analysis at 1 site and water-quality samples were collected at 7 springs, 21 wells, and 9 stream sites in the study area between November 2008 and December 2010. Continuous water-quality monitors were installed in three springs, two wells, and at one stream site. Three continuous stream-gaging stations were installed to measure gage height and a stagedischarge rating was developed at two of the three sites. Depth to water below land surface was continuously measured in two wells.
\end{abstract}

\section{Introduction}

The San Marcos Springs complex in Hays County, Tex. (fig. 1), is the second largest spring complex in Texas and a major discharge point for the Edwards aquifer (Brune, 1975) (fig. 1). The amount of discharge from the San Marcos Springs, together with groundwater altitude in the Bexar County index well (fig. 1) and discharge from the Comal Springs complex (fig. 1), serve as an indicator for enacting various water-management strategies in the San Antonio area (Texas Legislature Online, 2007). Stage 1 water restrictions are enforced in parts of the San Antonio area if the 10-day average altitude of water in the Bexar County index well is less than 660 feet above the National Geodetic Vertical Datum of 1929, the 10-day average discharge at the Comal Springs complex is less than 225 cubic feet per second $\left(\mathrm{ft}^{3} / \mathrm{s}\right)$, or the 10-day average discharge at the San Marcos Springs complex is less than $96 \mathrm{ft}^{3} / \mathrm{s}$. Under stage 1 water restrictions, municipal, industrial, and agricultural users of water from the Edwards aquifer in the San Antonio area are required to reduce withdrawals by 20 percent of their permitted allotment (Texas Legislature, 2007). Table 1 shows the conditions at the three indicator sites that correspond with the four stages of water restrictions. Because of the importance of San Marcos Springs as a water source and indicator for water management strategies, the U.S. Geological Survey (USGS), in cooperation with the San Antonio Water System (SAWS), collected geochemical and hydrologic data during 2008-10, in Bexar, Comal, and Hays Counties, Tex. (fig. 1), to define and characterize the sources of recharge to San Marcos Springs.

\section{Purpose and Scope}

This report presents the geochemical and hydrologic data collected during 2008-10 from 39 sites throughout Bexar, Comal, and Hays Counties (fig. 2). Stable isotopes measured in precipitation samples and selected physicochemical properties measured by using continuous water-quality monitors deployed at three springs, two wells, and one stream site also are presented. Hydrologic data include continuous gage height from three stream sites, continuous discharge from two stream sites, periodic discharge measurements made at two stream sites, and continuous depth of water below land surface from two wells.

\section{Study Area Description}

The San Antonio segment of the Edwards aquifer (hereinafter, Edwards aquifer) consists of Lower Cretaceous-age rocks of the Edwards Group (Rose, 1972) and the Georgetown Formation. Most recharge to the Edwards aquifer occurs in the recharge zone (aquifer outcrop) west of Bexar County (fig. 1), where streams originating north of the aquifer flow across the recharge zone and lose most, or all, of their flows into highly faulted and fractured limestone (Hanson and Small, 1995). Additional recharge enters the aquifer through the recharge zone in Bexar, Comal, and Hays Counties. After the water enters the aquifer in the area west of Bexar County, it generally moves in an easterly direction to discharge points in Bexar County, mainly municipal water-supply wells. Water not discharged to wells in Bexar County continues flowing towards the northeast along and parallel to northeast-trending 


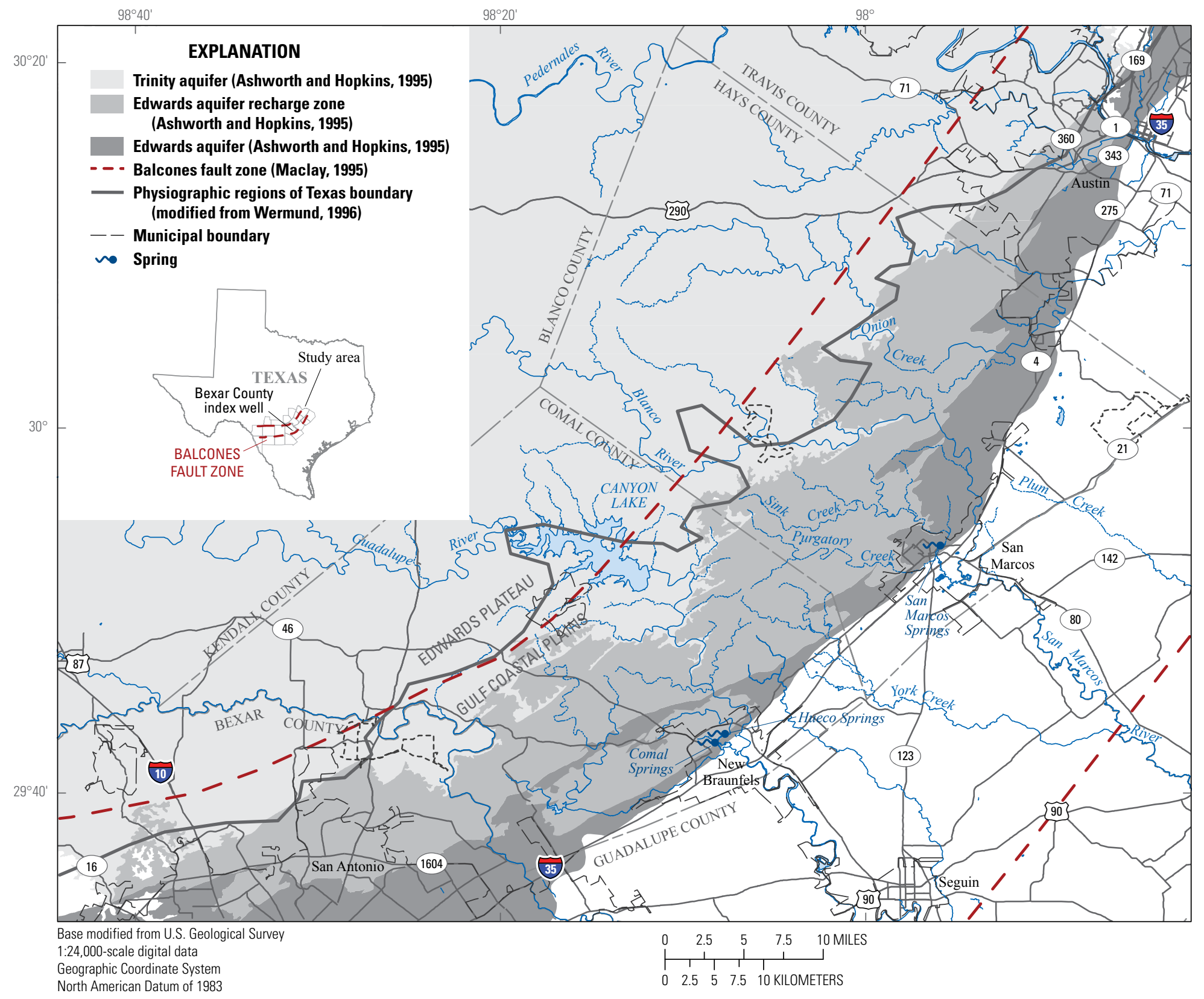

Figure 1. Study area for San Marcos Springs recharge study, Bexar, Comal, and Hays Counties, Texas. 
Table 1. Water restriction stage-threshold conditions at the three indicator sites for the Edwards aquifer, south-central Texas (Texas Legislature, 2007).

[NGVD29, National Geodetic Vertical Datum of 1929; <, less than; $\mathrm{ft}^{3} / \mathrm{s}$, cubic feet per second; N/A, not applicable]

\begin{tabular}{lcccc}
\hline $\begin{array}{c}\text { Indicator site } \\
\text { (based on 10-day average) }\end{array}$ & $\begin{array}{c}\text { Stage } \\
\mathbf{1}\end{array}$ & $\begin{array}{c}\text { Stage } \\
\mathbf{2}\end{array}$ & $\begin{array}{c}\text { Stage } \\
\mathbf{3}\end{array}$ & $\begin{array}{c}\text { Stage } \\
\mathbf{4}\end{array}$ \\
\hline $\begin{array}{l}\text { Bexar County index well level } \\
\text { (NGVD29) }\end{array}$ & $<660$ & $<650$ & $<640$ & $<630$ \\
$\begin{array}{l}\text { San Marcos Spring discharge } \\
\left(\mathrm{ft}^{3} / \mathrm{s}\right)\end{array}$ & $<96$ & $<80$ & $\mathrm{~N} / \mathrm{A}$ & $\mathrm{N} / \mathrm{A}$ \\
$\begin{array}{l}\left.\text { Comal Spring discharge ( } \mathrm{ft}^{3} / \mathrm{s}\right) \\
\begin{array}{l}\text { Withdrawal reductions for munic- } \\
\text { ipal, industrial, and agricultural } \\
\text { users (percent) }\end{array}\end{array}$ & 20 & 30 & 35 & 40 \\
\hline
\end{tabular}

faults in the study area to discharge points in Comal and Hays Counties, primarily Comal Springs in Comal County and San Marcos Springs in Hays County (Hanson and Small, 1995; Maclay and Land, 1988).

The study area is in an extensively faulted section of Cretaceous strata known as the Balcones fault zone (fig. 1). The fault zone developed as a result of extensional faulting and is characterized by a network of en-echelon, high-angle, mostly down-to-the-coast normal faults along the northwestern margin of the Gulf Coastal Plain (Maclay and Small, 1984; Maclay, 1995). The Cretaceous strata were vertically displaced, intensively fractured, and differentially rotated within a series of southwest-to-northeast trending fault blocks (Barker and Ardis, 1996). The fault blocks, and their subsequent erosion and dissolution, are major factors affecting flow in the aquifer.

The San Marcos Springs complex (fig. 3) issues from the San Marcos Springs fault (fig. 3), a normal fault with as much as 470 feet of offset (LBG-Guyton Associates, 2004) that juxtaposes the Edwards Group against the younger Austin and Taylor Groups. The long-term (May 1955-September 2010) average discharge $\left(174 \mathrm{ft}^{3} / \mathrm{s}\right)$ of the San Marcos Springs complex issues from well-defined orifices and numerous sand boils at the bottom of Spring Lake (fig. 3) (U.S. Geological Survey, 2010). LBG-Guyton Associates (2004) estimated that about 25 percent of the total discharge from San Marcos Springs emerges from well-defined orifices and the remaining 75 percent from sand boils. The water discharging from San Marcos Springs is impounded in Spring Lake and discharges over two separate outflow structures (west and east outflows; fig. 3). The west outflow channel spills over a series of waterfalls with adjustable gates that can control the water-surface elevation in Spring Lake and, subsequently, the rate of discharge out of the lake. The east overflow channel is controlled by a fixed concrete structure. The two channels converge below the outflow structures as the headwaters of the
San Marcos River. The concrete dam at the outlet of Spring Lake is located between the two outflow structures and likely allows a substantial amount of underflow (fig. 3) (Gary and others, 2008).

Water that discharges from the San Marcos Springs complex is believed to be a mixture of regional groundwater that entered the Edwards aquifer west of Bexar County and local groundwater that entered the aquifer in proximity to the San Marcos Springs complex (Puente, 1976; Ogden and others, 1986; Maclay, 1995; LBG-Guyton Associates, 2004). Studies by Ogden and others (1986) and Johnson and Schindel (2008) indicate certain spring orifices at the complex might be sourced by regional groundwater while other discharge points might be sourced by local groundwater.

\section{Site Information}

The USGS collected water-quality and hydrologic data from nine surface-water sites on five streams (Guadalupe River, Sink Creek, Purgatory Creek, Blanco River, and Cibolo Creek) during the study period (fig. 1; table 2). The Guadalupe and Blanco Rivers are perennial streams and Sink, Purgatory, and Cibolo Creeks are ephemeral streams. Six surface-water sites were located on the perennial streams and three were located on the ephemeral streams (fig. 2; table 2).

Water-quality and hydrologic data were collected from a total of 22 wells from November 2008-December 2010 (fig. 2; table 3). Nine of the wells were public supply wells, seven were domestic supply wells, two supplied water for livestock, three were monitoring wells (wells used only for monitoring water levels and collecting water-quality samples), and one supplied an institutional user. Seventeen of the 22 wells were completed entirely in the Edwards aquifer and four were completed entirely in the Trinity aquifer. The remaining well was completed so that both the Edwards aquifer and the Trinity aquifer contributed water to the well. The depths of the 22 wells ranged from 32.5 feet to 732 feet below land surface. The tops of the open intervals in the wells ranged in depth from land surface to 580 feet below land surface and the bottoms of the open intervals ranged in depth from 32.5 to 732 feet below land surface.

Water-quality and hydrologic data were collected from seven springs during November 2008-December 2010 (table 3). The seven springs included three spring orifices in the Comal Springs complex, one spring orifice in the Hueco Springs complex, and three spring orifices in the San Marcos Springs complex.

\section{Methods}

Physicochemical properties (dissolved oxygen concentration, $\mathrm{pH}$, specific conductance, water temperature, and turbidity) and constituents (total dissolved solids [TDS], major ions, nutrients, trace elements, dissolved organic carbon [DOC], 


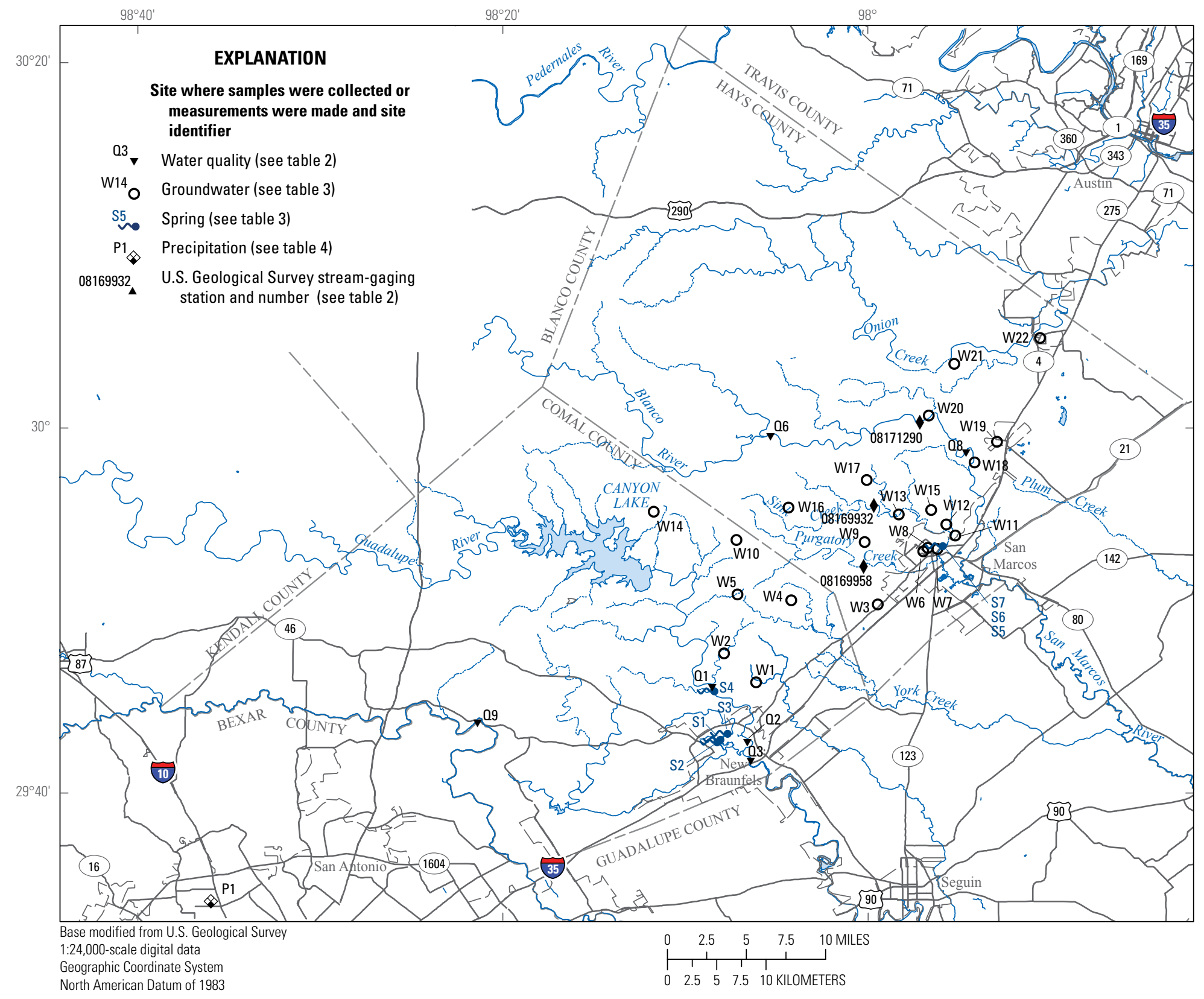

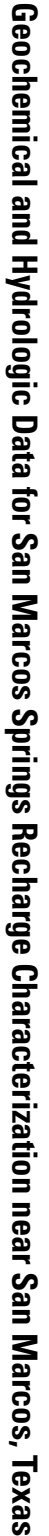

Figure 2. Locations of spring, stream, groundwater, and precipitation sites for San Marcos Springs recharge study, Bexar, Comal, and Hays Counties, Texas. 


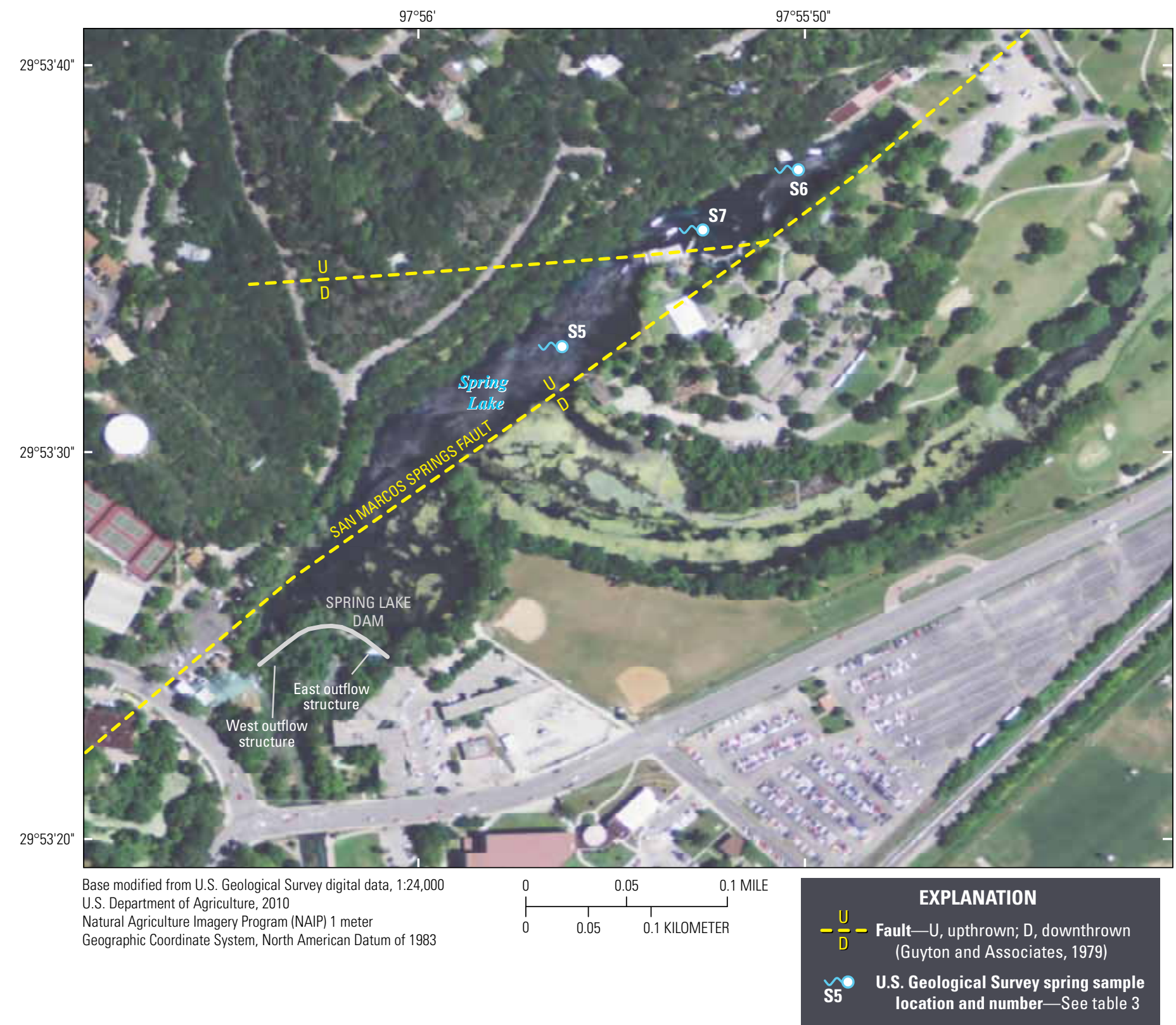

Figure 3. San Marcos Springs complex, Hays County, Texas. 
Table 2. Surface-water sites from which data were collected for the San Marcos Springs recharge study, Bexar, Comal, and Hays Counties, Texas.

[fig., figure; dd, degrees; mm, minutes; ss, seconds; USGS, U.S. Geological Survey; NAD83, North American Datum of 1983; NAVD88, North American Vertical Datum of 1988; QW, periodic water-quality samples; G, continuous gage height; Q, continuous discharge; M, continuous water-quality monitor]

\begin{tabular}{|c|c|c|c|c|c|c|c|}
\hline $\begin{array}{c}\text { Map } \\
\text { identi- } \\
\text { fier } \\
\text { (fig. 2) }\end{array}$ & $\begin{array}{l}\text { USGS } \\
\text { station } \\
\text { number }\end{array}$ & USGS station name & $\begin{array}{c}\text { Site } \\
\text { identifier }\end{array}$ & $\begin{array}{l}\text { Latitude, in } \\
\text { dd mm ss } \\
\text { (NAD83) }\end{array}$ & $\begin{array}{l}\text { Longitude, in } \\
\text { dd mm ss } \\
\text { (NAD83) }\end{array}$ & $\begin{array}{c}\text { Altitude } \\
\text { of land } \\
\text { surface } \\
\text { (NAVD88) }\end{array}$ & $\begin{array}{l}\text { Data } \\
\text { type(s) }\end{array}$ \\
\hline Q1 & 08167990 & $\begin{array}{l}\text { Guadalupe River at River Road near } \\
\text { Sattler, Tex. }\end{array}$ & $\begin{array}{l}\text { Guadalupe at River } \\
\text { Road }\end{array}$ & $29^{\circ} 45^{\prime} 55^{\prime \prime}$ & $98^{\circ} 08^{\prime} 31^{\prime \prime}$ & 640 & QW \\
\hline Q2 & 08168500 & $\begin{array}{l}\text { Guadalupe River above Comal River at } \\
\text { New Braunfels, Tex. }\end{array}$ & $\begin{array}{l}\text { Guadalupe above } \\
\text { Comal }\end{array}$ & $29^{\circ} 42^{\prime} 54^{\prime \prime}$ & $98^{\circ} 06^{\prime} 36^{\prime \prime}$ & 587 & QW \\
\hline Q3 & 08169500 & Guadalupe River at New Braunfels, Tex. & $\begin{array}{l}\text { Guadalupe at New } \\
\text { Braunfels }\end{array}$ & $29^{\circ} 41^{\prime} 53^{\prime \prime}$ & $98^{\circ} 06^{\prime} 24^{\prime \prime}$ & 572.9 & QW \\
\hline Q4 & 08169932 & Sink Creek near San Marcos, Tex. & Sink Creek & $29^{\circ} 55^{\prime} 45.57^{\prime \prime}$ & 9759'39.33" & 742 & QW, G, Q \\
\hline Q5 & 08169958 & $\begin{array}{l}\text { Purgatory Creek at Mountain High Drive } \\
\text { near San Marcos, Tex. }\end{array}$ & Purgatory Creek & $29^{\circ} 52^{\prime} 21.12^{\prime \prime}$ & $98^{\circ} 00^{\prime} 14.1^{\prime \prime}$ & 690 & QW, G \\
\hline Q6 & 08171000 & Blanco River at Wimberley, Tex. & Blanco at Wimberley & $29^{\circ} 59^{\prime} 40^{\prime \prime}$ & $98^{\circ} 05^{\prime} 20^{\prime \prime}$ & 797.6 & QW \\
\hline Q7 & 08171290 & $\begin{array}{l}\text { Blanco River at Halifax Ranch near Kyle, } \\
\text { Tex. }\end{array}$ & Blanco at Halifax & $30^{\circ} 00^{\prime} 20^{\prime \prime}$ & $97^{\circ} 57^{\prime} 09^{\prime \prime}$ & 675 & $\begin{array}{l}\text { QW, G, } \\
\text { Q, M }\end{array}$ \\
\hline Q8 & 08171300 & Blanco River near Kyle, Tex. & Blanco near Kyle & $29^{\circ} 58^{\prime} 46^{\prime \prime}$ & 9754'36" & 620.5 & QW \\
\hline Q9 & 08184300 & $\begin{array}{l}\text { Cibolo Creek at Farm Road } 1863 \text { below } \\
\text { Bulverde, Tex. }\end{array}$ & Cibolo Creek & $29^{\circ} 43^{\prime} 57.6^{\prime \prime}$ & $98^{\circ} 21^{\prime} 22^{\prime \prime}$ & 941 & QW \\
\hline
\end{tabular}

total organic carbon [TOC], and select stable isotopes) were measured in water-quality samples collected periodically at sites throughout the study area. Periodic water-quality samples were collected at 9 stream sites, 21 wells, and 7 springs in the study area during November 2008-December 2010. Precipitation samples were collected at one site (P1, fig. 2) and analyzed for stable isotopes. In addition to periodic waterquality sampling, continuous water-quality monitors were installed at one stream site, in two wells, and in three springs. Water-surface elevations (gage heights) were measured every 15 minutes from stage sensors at each of three gaged stream sites (fig. 2; table 2). A stage-discharge rating was developed for two of the gaged stream sites to compute continuous (every 15 minute) discharge data. The depth of groundwater below land surface was continuously measured in the two wells in which water-quality monitors were installed.

\section{Periodic Water-Quality Samples}

Using a mixture of periodic (routine) samples and samples collected in response to storms, 305 environmental water-quality samples (appendix 1) and 32 quality-assurance samples (appendixes 1-2) were collected. The samples were collected over the course of 20 sampling events (hereinafter, sampling cycles) during November 2008-December 2010 (table 4). The 20 sampling cycles included 17 routine and 3 storm sampling cycles (sampling cycles 8, 9, and 17). Thirty sites were sampled during the first sampling cycle. Of these 30 sites, 15 sites were selected for long-term sampling based on site type, water quality, aquifer, and location. The 15 sites selected for long-term sampling (sampling identifier LT, table 4) included two surface-water sites, nine wells, and four springs. Two of the nine wells (sampling identifier R, table 4) had to be replaced within the first 2 months of longterm sampling because of water-quality and mechanical problems. These wells were replaced with two monitoring wells (sampling identifier LT2, table 4) located on either side of the San Marcos Springs fault. Well LT-67-09-113 (hereinafter, Fish Hatchery; fig. 2, map identifier W3; table 3), located in the downthrown block of the fault, was sampled quarterly because historical data showed the water chemistry in this well was stable. Well LR-58-57-512 (hereinafter, Ruby Ranch; fig. 2, map identifier W21) was not sampled beyond sampling cycle 13 because the well was permanently removed from service by the water purveyor. The Ruby Ranch well was not replaced and three additional wells (sampling identifier R2, table 4) were no longer sampled beginning in sampling cycle 15 to redistribute resources in case there was an additional storm event that could be sampled. Spring LR-67-01-820 (hereinafter, Weissmuller Spring; fig. 2, map identifier S6; table 3) also was added to the long-term sampling sites beginning with sampling cycle 15 , bringing the number of periodically sampled sites to 12 . The final 12 sites (sampling identifier F, table 4) were sampled through December 2010 . 
Table 3. Wells and springs from which data were collected for the San Marcos Springs recharge study, Comal and Hays Counties, Texas.

[fig., figure; USGS, U.S. Geological Survey; dd, degrees; mm, minutes; ss, seconds;NAD83, North American Datum of 1983; NAVD88, North American Vertical Datum of 1988; N/A, not applicable; X, open hole; W, walled, S, Screened; QW, periodic water-quality samples; WL, continuous groundwater altitude; M, continuous water-quality monitor; --, not available]

\begin{tabular}{|c|c|c|c|c|c|c|c|c|c|c|c|}
\hline $\begin{array}{l}\text { Map } \\
\text { identi- } \\
\text { fier } \\
\text { (fig. 2) }\end{array}$ & $\begin{array}{c}\text { USGS station } \\
\text { number }\end{array}$ & $\begin{array}{l}\text { State well } \\
\text { number }\end{array}$ & $\begin{array}{c}\text { Site } \\
\text { identifier }\end{array}$ & $\begin{array}{l}\text { Latitude, } \\
\text { in dd mm ss } \\
\text { (NAD83) }\end{array}$ & $\begin{array}{l}\text { Longitude, } \\
\text { in dd mm ss } \\
\text { (NAD83) }\end{array}$ & $\begin{array}{c}\text { Altitude } \\
\text { of land } \\
\text { surface } \\
\text { (NAVD88) }\end{array}$ & $\begin{array}{c}\text { Contributing } \\
\text { aquifer(s) }\end{array}$ & $\begin{array}{l}\text { Total } \\
\text { depth } \\
\text { (feet) }\end{array}$ & $\begin{array}{c}\text { Open } \\
\text { interval } \\
\text { (feet) }\end{array}$ & $\begin{array}{c}\text { Comple- } \\
\text { tion } \\
\text { type }\end{array}$ & $\begin{array}{l}\text { Data } \\
\text { type(s) }\end{array}$ \\
\hline \multicolumn{12}{|c|}{ Wells } \\
\hline W1 & 294604098060701 & DX-68-16-707 & $4 \mathrm{D}$ & -- & -- & 802 & Edwards & 400 & $195-400$ & $\mathrm{X}$ & QW \\
\hline W2 & 294739098075301 & DX-68-15-605 & Bonem & $29^{\circ} 47^{\prime} 39^{\prime \prime}$ & $98^{\circ} 07^{\prime} 53^{\prime \prime}$ & 860 & Edwards & 375 & $220-375$ & $\mathrm{~W}$ & QW \\
\hline W3 & 295019097592701 & LR-67-09-113 & Fish Hatchery & $29^{\circ} 50^{\prime} 19.46^{\prime \prime}$ & $97^{\circ} 59^{\prime} 26.65^{\prime \prime}$ & 714 & Edwards & 280 & $216-280$ & $\mathrm{X}$ & QW \\
\hline W4 & 295033098041201 & DX-68-16-201 & Mendez & $29^{\circ} 50^{\prime} 33^{\prime \prime}$ & $98^{\circ} 04^{\prime} 12^{\prime \prime}$ & 991 & Trinity & 640 & $580-640$ & $\mathrm{~S}$ & QW \\
\hline W5 & 295052098070801 & DX-68-16-101 & Sac-N-Pac & $29^{\circ} 50^{\prime} 52^{\prime \prime}$ & $98^{\circ} 07^{\prime} 08^{\prime \prime}$ & 1,020 & Trinity & 408 & $256-408$ & $\mathrm{X}$ & QW \\
\hline W6 & 295314097565701 & LR-67-01-826 & TSU - West Campus & $29^{\circ} 53^{\prime} 14.04^{\prime \prime}$ & $97^{\circ} 56^{\prime} 57.47^{\prime \prime}$ & 751 & Edwards & 210 & $103-210$ & $\mathrm{X}$ & QW, WL, M \\
\hline W7 & 295323097561101 & LR-67-01-828 & TSU - Artesian & $29^{\circ} 53^{\prime} 22.5^{\prime \prime}$ & $97^{\circ} 56^{\prime} 11.2^{\prime \prime}$ & 577 & Edwards & 600 & 0-199 & $\mathrm{X}$ & QW \\
\hline W8 & 295325097564301 & LR-67-01-827 & TSU - Jackson & $29^{\circ} 53^{\prime} 25^{\prime \prime}$ & $97^{\circ} 56^{\prime} 42.9^{\prime \prime}$ & 740 & Edwards & 191 & $135-191$ & $\mathrm{X}$ & QW \\
\hline W9 & 295345098001001 & LR-68-08-902 & SMBA & $29^{\circ} 53^{\prime} 45^{\prime \prime}$ & $98^{\circ} 00^{\prime} 10^{\prime \prime}$ & 770 & Edwards & 335 & $200-335$ & $\mathrm{X}$ & QW \\
\hline W10 & 295352098071201 & DX-68-08-701 & Riedel & $29^{\circ} 53^{\prime} 52^{\prime \prime}$ & $98^{\circ} 07^{\prime} 12^{\prime \prime}$ & 1,150 & Edwards & 240 & $239-240$ & $\mathrm{~S}$ & QW \\
\hline W11 & 295406097551201 & LR-67-01-818 & Horton & $29^{\circ} 54^{\prime} 06^{\prime \prime}$ & $97^{\circ} 55^{\prime} 12^{\prime \prime}$ & 610 & Edwards & 80 & $10-80$ & $\mathrm{X}$ & QW \\
\hline W12 & 295443097554201 & LR-67-01-809 & Tipps & $29^{\circ} 54^{\prime} 43^{\prime \prime}$ & $97^{\circ} 55^{\prime} 42^{\prime \prime}$ & 601.3 & Edwards & 32.5 & $0-32.5$ & $\mathrm{~W}$ & WL,M \\
\hline W13 & 295515097581801 & LR-67-01-403 & Solar & $29^{\circ} 55^{\prime} 15.1^{\prime \prime}$ & $97^{\circ} 58^{\prime} 17.9^{\prime \prime}$ & 688 & Edwards & -- & -- & $\mathrm{X}$ & QW \\
\hline W14 & 295524098114401 & DX-68-07-505 & Eagle Peak & -- & -- & 1,177 & Trinity & 732 & $200-732$ & $\mathrm{X}$ & QW \\
\hline W15 & 295530097563201 & LR-67-01-503 & Neff & $29^{\circ} 55^{\prime} 30^{\prime \prime}$ & $97^{\circ} 56^{\prime} 32^{\prime \prime}$ & 733 & Edwards & 280 & $\begin{array}{l}180-240 \\
240-280\end{array}$ & $\begin{array}{l}\mathrm{S} \\
\mathrm{X}\end{array}$ & QW \\
\hline W16 & 295538098042101 & LR-68-08-502 & Burns & $29^{\circ} 55^{\prime} 38^{\prime \prime}$ & $98^{\circ} 04^{\prime} 21^{\prime \prime}$ & 1,041 & Trinity & 700 & $400-700$ & $\mathrm{~S}$ & QW \\
\hline W17 & 295709098000301 & LR-68-08-602 & Laguna & $29^{\circ} 57^{\prime} 08.5^{\prime \prime}$ & $98^{\circ} 00^{\prime} 03^{\prime \prime}$ & 906 & Edwards & 600 & -- & $\mathrm{X}$ & QW \\
\hline W18 & 295806097540901 & LR-67-01-312 & Aqua Texas & -- & -- & 683 & Edwards & 520 & $300-520$ & $\mathrm{X}$ & QW \\
\hline W19 & 295915097525501 & LR-67-01-309 & City of Kyle & -- & -- & 753 & Edwards & 658 & $328-658$ & $\mathrm{X}$ & QW \\
\hline W20 & 300041097563901 & LR-58-57-808 & Halifax & $30^{\circ} 00^{\prime} 41^{\prime \prime}$ & $97^{\circ} 56^{\prime} 39^{\prime \prime}$ & 740 & Edwards & 220 & $161-220$ & $\mathrm{~W}$ & QW \\
\hline W21 & 300331097551601 & LR-58-57-512 & Ruby Ranch & -- & -- & 830 & Edwards/Trinity & 405 & $182-405$ & $\mathrm{X}$ & QW \\
\hline W22 & 300453097503301 & LR-58-58-403 & City of Buda & -- & -- & 710 & Edwards & 390 & $168-390$ & $\mathrm{X}$ & QW \\
\hline \multicolumn{12}{|c|}{ Springs } \\
\hline S1 & 294255098080501 & DX-68-23-324 & Comal 3 Spring & $29^{\circ} 42^{\prime} 55.38^{\prime \prime}$ & $98^{\circ} 08^{\prime} 04.92^{\prime \prime}$ & 625 & Edwards & N/A & $\mathrm{N} / \mathrm{A}$ & $\mathrm{N} / \mathrm{A}$ & QW \\
\hline S2 & 294300098080001 & DX-68-23-301 & Comal Spring & $29^{\circ} 42^{\prime} 46^{\prime \prime}$ & $98^{\circ} 08^{\prime} 15^{\prime \prime}$ & 623.43 & Edwards & N/A & N/A & $\mathrm{N} / \mathrm{A}$ & QW \\
\hline S3 & 294314098074101 & DX-68-23-326 & Comal 5 Spring & $29^{\circ} 43^{\prime} 14.22^{\prime \prime}$ & $98^{\circ} 07^{\prime} 41.46^{\prime \prime}$ & 626 & Edwards & $\mathrm{N} / \mathrm{A}$ & $\mathrm{N} / \mathrm{A}$ & N/A & QW \\
\hline S4 & 294533098082301 & DX-68-15-901 & Hueco Spring & $29^{\circ} 45^{\prime} 34^{\prime \prime}$ & $98^{\circ} 08^{\prime} 24^{\prime \prime}$ & 652.53 & Edwards & $\mathrm{N} / \mathrm{A}$ & $\mathrm{N} / \mathrm{A}$ & N/A & QW \\
\hline S5 & 295322097561000 & LR-67-01-819 & Deep Spring & $29^{\circ} 53^{\prime} 33^{\prime \prime}$ & $97^{\circ} 55^{\prime} 54^{\prime \prime}$ & 600 & Edwards & N/A & $\mathrm{N} / \mathrm{A}$ & $\mathrm{N} / \mathrm{A}$ & QW,M \\
\hline S6 & 295322097561002 & LR-67-01-820 & Weissmuller Spring & $29^{\circ} 53^{\prime} 36^{\prime \prime}$ & $97^{\circ} 55^{\prime} 48^{\prime \prime}$ & 600 & Edwards & $\mathrm{N} / \mathrm{A}$ & $\mathrm{N} / \mathrm{A}$ & N/A & QW,M \\
\hline S7 & 295336097555201 & LR-67-01-825 & Diversion Spring & $29^{\circ} 53^{\prime} 35.64^{\prime \prime}$ & $97^{\circ} 55^{\prime} 51.9^{\prime \prime}$ & 580 & Edwards & N/A & $\mathrm{N} / \mathrm{A}$ & $\mathrm{N} / \mathrm{A}$ & QW,M \\
\hline
\end{tabular}


Table 4. Sampling cycles and number of samples collected during each cycle (environmental and quality assurance), San Marcos Springs recharge study, Bexar, Comal, and Hays Counties, Texas.

[a, not selected for long-term sampling; --, not sampled; b, preceding storm sampling occurred during routine scheduled sample; i, one sample for stable isotopes only; D, duplicate; B, blank; c, well not accessible; g, sampling discontinued because of water-quality issues; $x$, well taken out of service; $p$, sampling discontinued because of pump problems at well; I, sampled only as part of the initial 30 sites; LT, sampled as one of initial 15 long-term sampling sites; LT2, substituted for one of 15 long-term sampling sites; F, sampled as one of the final 12 sites; S, sampled only during storms; R, removed from long-term sampling; R2, removed from long-term sampling in sampling cycle 15; P, precipitation samples]

\begin{tabular}{|c|c|c|c|c|c|c|c|c|c|c|c|}
\hline \multirow{3}{*}{$\begin{array}{c}\text { Map } \\
\text { identi- } \\
\text { fier } \\
\text { (fig. 2) }\end{array}$} & \multirow{3}{*}{$\begin{array}{c}\text { USGS } \\
\text { station number }\end{array}$} & \multirow{3}{*}{$\begin{array}{c}\text { Site } \\
\text { identifier }\end{array}$} & \multicolumn{9}{|c|}{ Sampling cycles } \\
\hline & & & 1 & 2 & 3 & 4 & 5 & 6 & 7 & 8 & 9 \\
\hline & & & $\begin{array}{l}\text { Dec. } \\
2008\end{array}$ & $\begin{array}{l}\text { Feb.- } \\
\text { Mar. } \\
2009\end{array}$ & $\begin{array}{l}\text { Mar. } \\
2009\end{array}$ & $\begin{array}{l}\text { Apr. } \\
2009\end{array}$ & $\begin{array}{c}\text { May- } \\
\text { June } \\
2009\end{array}$ & $\begin{array}{l}\text { July } \\
2009\end{array}$ & $\begin{array}{l}\text { Aug.- } \\
\text { Sept. } \\
2009\end{array}$ & $\begin{array}{c}\text { Sept. } \\
2009 \\
\text { (storm) }\end{array}$ & $\begin{array}{c}\text { Oct. } \\
2009 \\
\text { (storm) }\end{array}$ \\
\hline \multicolumn{12}{|c|}{ Surface-water sites } \\
\hline Q1 & 08167990 & Guadalupe at River Road & 1 & 1 & 1 & 1 & 1 & 1 & 1 & 1 & 1 \\
\hline Q4 & 08169932 & Sink Creek & -- & -- & -- & -- & -- & -- & -- & -- & 1 \\
\hline Q5 & 08169958 & Purgatory Creek & -- & -- & -- & -- & -- & -- & -- & -- & -- \\
\hline Q6 & 08171000 & Blanco at Wimberley & $1 \mathrm{a}$ & -- & -- & -- & -- & -- & -- & -- & -- \\
\hline Q7 & 08171290 & Blanco at Halifax & 1 & 1 & 1 & 1 & $1 \mathrm{D}$ & 1 & $1 \mathrm{D}$ & 2 & 1 \\
\hline Q8 & 08171300 & Blanco near Kyle & -- & -- & -- & -- & -- & -- & -- & -- & -- \\
\hline W2 & 294739098075301 & Bonem & $1 \mathrm{a}$ & -- & -- & -- & -- & -- & -- & -- & -- \\
\hline W3 & 295019097592701 & Fish Hatchery & -- & -- & -- & 1 & -- & $1 \mathrm{D}$ & 1 & -- & -- \\
\hline W4 & 295033098041201 & Mendez & 1 & 1 & 1 & 1 & 1 & 1 & 1 & -- & -- \\
\hline W5 & 295052098070801 & Sac-N-Pac & 1 & 1 & 1 & 1 & 1 & 1 & 1 & -- & -- \\
\hline W6 & 295314097565701 & TSU - West Campus & -- & -- & -- & -- & 1 & 1 & 1 & -- & -- \\
\hline W7 & 295323097561101 & TSU - Artesian & $1 \mathrm{a}$ & -- & -- & -- & -- & -- & -- & -- & -- \\
\hline W8 & 295325097564301 & TSU - Jackson & 1a & -- & -- & -- & -- & -- & -- & -- & -- \\
\hline W9 & 295345098001001 & SMBA & $1 \mathrm{a}$ & -- & -- & -- & -- & -- & -- & -- & -- \\
\hline W10 & 295352098071201 & Riedel & $1 \mathrm{aD}$ & -- & -- & -- & -- & -- & -- & -- & -- \\
\hline W11 & 295406097551201 & Horton & $1 \mathrm{a}$ & -- & -- & -- & -- & -- & -- & -- & -- \\
\hline W20 & 300041097563901 & Halifax & 1a & -- & -- & -- & -- & -- & -- & -- & -- \\
\hline W21 & 300331097551601 & Ruby Ranch & 1 & $1 \mathrm{D}$ & 1 & 1 & 1 & 1 & $\mathrm{c}$ & -- & -- \\
\hline W22 & 300453097503301 & City of Buda & 1 & $1 p$ & -- & -- & -- & -- & -- & -- & -- \\
\hline \multicolumn{12}{|c|}{ Springs } \\
\hline S1 & 294255098080501 & Comal 3 Spring & $1 \mathrm{a}$ & -- & -- & -- & -- & -- & -- & -- & -- \\
\hline S2 & 294300098080001 & Comal Spring & 1 & 1 & 1 & 1 & 1 & 1 & 1 & 1 & 1 \\
\hline $\mathrm{S} 3$ & 294314098074101 & Comal 5 Spring & 1a & -- & -- & -- & -- & -- & -- & -- & -- \\
\hline S4 & 294533098082301 & Hueco Spring & 1 & 1 & 1 & $1 \mathrm{~B}$ & 1 & 1 & 1 & 1 & 1 \\
\hline S5 & 295322097561000 & Deep Spring & 1 & 1 & 1 & 1 & 1 & 1 & 1 & 5 & 6 \\
\hline S6 & 295322097561002 & Weissmuller Spring & -- & -- & -- & -- & -- & -- & -- & -- & -- \\
\hline S7 & 295336097555201 & Diversion Spring & 1B & 1 & 1 & 1 & 1 & 1 & 1 & 5 & 6 \\
\hline \multicolumn{12}{|c|}{ Precipitation } \\
\hline P1 & 293146982941 & USGS San Antonio & -- & -- & -- & -- & -- & -- & -- & -- & -- \\
\hline
\end{tabular}


Table 4. Sampling cycles and number of samples collected during each cycle (environmental and quality assurance), San Marcos Springs recharge study, Bexar, Comal, and Hays Counties, Texas.-Continued

[a, not selected for long-term sampling; --, not sampled; b, preceding storm sampling occurred during routine scheduled sample; i, one sample for stable isotopes only; D, duplicate; B, blank; c, well not accessible; g, sampling discontinued because of water-quality issues; $x$, well taken out of service; $p$, sampling discontinued because of pump problems at well; I, sampled only as part of the initial 30 sites; LT, sampled as one of initial 15 long-term sampling sites; LT2, substituted for one of 15 long-term sampling sites; F, sampled as one of the final 12 sites; S, sampled only during storms; R, removed from long-term sampling; R2, removed from long-term sampling in sampling cycle 15; $\mathrm{P}$, precipitation samples]

\begin{tabular}{|c|c|c|c|c|c|c|c|c|c|c|c|c|c|c|}
\hline \multirow{3}{*}{$\begin{array}{c}\text { Map } \\
\text { identi- } \\
\text { fier } \\
\text { (fig. 2) }\end{array}$} & \multicolumn{11}{|c|}{ Sampling cycles } & \multirow{3}{*}{$\begin{array}{c}\text { Routine } \\
\text { sam- } \\
\text { ples }\end{array}$} & \multirow{3}{*}{$\begin{array}{c}\text { Storm } \\
\text { sam- } \\
\text { ples }\end{array}$} & \multirow{3}{*}{$\begin{array}{l}\text { Sam- } \\
\text { pling } \\
\text { identifier }\end{array}$} \\
\hline & 10 & 11 & 12 & 13 & 14 & 15 & 16 & 17 & 18 & 19 & 20 & & & \\
\hline & $\begin{array}{l}\text { Oct. } \\
2009\end{array}$ & $\begin{array}{l}\text { Nov. } \\
2009\end{array}$ & $\begin{array}{l}\text { Dec. } \\
2009\end{array}$ & $\begin{array}{l}\text { Jan. } \\
2010\end{array}$ & $\begin{array}{l}\text { Feb. } \\
2010\end{array}$ & $\begin{array}{l}\text { June } \\
2010\end{array}$ & $\begin{array}{l}\text { Aug. } \\
2010\end{array}$ & $\begin{array}{c}\text { Sept. } \\
2010 \\
\text { (storm) }\end{array}$ & $\begin{array}{c}\text { Sept.- } \\
\text { Oct. } \\
2010 \\
\end{array}$ & $\begin{array}{l}\text { Oct. } \\
2010\end{array}$ & $\begin{array}{l}\text { Nov.- } \\
\text { Dec. } \\
2010\end{array}$ & & & \\
\hline \multicolumn{15}{|c|}{ Surface-water sites } \\
\hline Q1 & $\mathrm{b}$ & 1 & 1 & 1 & 1 & 1 & 1 & -- & 1 & 1 & 1 & 16 & 2 & LT,F \\
\hline Q4 & -- & -- & -- & -- & -- & -- & -- & $1 \mathrm{~B}$ & -- & -- & -- & 0 & 2 & $\mathrm{~S}$ \\
\hline Q5 & -- & -- & -- & -- & -- & -- & -- & 1 & -- & -- & -- & 0 & 1 & $\mathrm{~S}$ \\
\hline Q6 & -- & -- & -- & -- & -- & -- & -- & -- & -- & -- & -- & 1 & 0 & $\mathrm{I}$ \\
\hline Q7 & $\mathrm{b}$ & 1 & 1 & 1 & 1 & 1 & 1 & 1 & $1 \mathrm{D}$ & 1 & 1 & 16 & 4 & LT,F \\
\hline Q8 & -- & -- & -- & -- & -- & -- & -- & 2 & -- & -- & -- & 0 & 2 & $\mathrm{~S}$ \\
\hline W2 & -- & -- & -- & -- & -- & -- & -- & -- & -- & -- & -- & 1 & 0 & I \\
\hline W3 & -- & -- & -- & 1 & -- & -- & -- & -- & -- & -- & -- & 4 & 0 & $\mathrm{LT} 2, \mathrm{R} 2$ \\
\hline W4 & 1 & 1 & 1 & 1 & 1 & 1 & 1 & -- & 1 & 1 & 1 & 17 & 0 & LT, F \\
\hline W5 & 1 & 1 & 1 & 1 & 1 & -- & -- & -- & -- & -- & -- & 12 & 0 & LT, R2 \\
\hline W6 & $\mathrm{c}$ & $1 \mathrm{~B}$ & $1 \mathrm{D}$ & 1 & 1 & $1 \mathrm{D}$ & 1 & -- & 1 & 1 & $1 \mathrm{~B}$ & 12 & 0 & LT2, F \\
\hline W7 & -- & -- & -- & -- & -- & -- & -- & -- & -- & -- & -- & 1 & 0 & I \\
\hline W8 & -- & -- & -- & -- & -- & -- & -- & -- & -- & -- & -- & 1 & 0 & I \\
\hline W9 & -- & -- & -- & -- & -- & -- & -- & -- & -- & -- & -- & 1 & 0 & I \\
\hline W10 & -- & -- & -- & -- & -- & -- & -- & -- & -- & -- & -- & 1 & 0 & I \\
\hline W11 & -- & -- & -- & -- & -- & -- & -- & -- & -- & -- & -- & 1 & 0 & I \\
\hline W20 & -- & -- & -- & -- & -- & -- & -- & -- & -- & -- & -- & 1 & 0 & I \\
\hline W21 & $\mathrm{c}$ & 1 & 1 & $1 x$ & -- & -- & -- & -- & -- & -- & -- & 9 & 0 & LT \\
\hline W22 & -- & -- & -- & -- & -- & -- & -- & -- & -- & -- & -- & 2 & 0 & $\mathrm{LT}, \mathrm{R}$ \\
\hline \multicolumn{15}{|c|}{ Springs } \\
\hline S1 & -- & -- & -- & -- & -- & -- & -- & -- & -- & -- & -- & 1 & 0 & I \\
\hline S2 & $\mathrm{b}$ & 1 & 1 & 1 & 1 & $2 \mathrm{i}$ & 1 & $7 \mathrm{D}$ & $\mathrm{b}$ & 1 & 1 & 16 & 9 & $\mathrm{LT}, \mathrm{F}$ \\
\hline S3 & -- & -- & -- & -- & -- & -- & -- & -- & -- & -- & -- & 1 & 0 & I \\
\hline S4 & $\mathrm{b}$ & 1 & 1 & 1 & 1 & $2 \mathrm{i}$ & 1 & $7 \mathrm{D}$ & $\mathrm{b}$ & 1 & 1 & 16 & 9 & LT, F \\
\hline S5 & $\mathrm{b}$ & $1 \mathrm{D}$ & 1 & 1 & 1 & 1 & $1 \mathrm{D}$ & 7 & $\mathrm{~b}$ & 1 & 1 & 15 & 18 & $\mathrm{LT}, \mathrm{F}$ \\
\hline S6 & -- & -- & -- & -- & -- & 1 & 1 & $7 \mathrm{D}$ & $\mathrm{b}$ & 1 & 1 & 4 & 7 & $\mathrm{~F}$ \\
\hline S7 & $\mathrm{b}$ & 1 & 1 & 1 & 1 & 1 & 1 & 7 & $\mathrm{~b}$ & 1 & 1 & 15 & 18 & LT, F \\
\hline \multicolumn{15}{|c|}{ Precipitation } \\
\hline $\mathrm{P} 1$ & -- & -- & & -- & -- & -- & -- & -- & -- & -- & -- & 0 & 24 & $\mathrm{P}$ \\
\hline
\end{tabular}




\section{Sample Collection}

Water-quality sample collection and processing followed standard USGS methods documented in the "National Field Manual for the Collection of Water-Quality Data" (U.S. Geological Survey, variously dated). During base flow, depthintegrated samples were collected using a Teflon bottle and nozzle, either by using multiple verticals when stream velocities were less than approximately 1.5 feet per second ( $\mathrm{ft} / \mathrm{s})$ or by using the equal-width increment method when stream velocities were greater than approximately $1.5 \mathrm{ft} / \mathrm{s}$. Automatic samplers were installed to collect water-quality samples during storm events at USGS stream-gaging stations 08169932 Sink Creek near San Marcos, Tex. (hereinafter, Sink Creek; fig. 2, map identifier Q4), and 08169958 Purgatory Creek at Mountain High Drive near San Marcos, Tex. (hereinafter, Purgatory Creek; fig. 2, map identifier Q5). When a predetermined stage was detected by the stream-gaging instrumentation, the automatic sampler was activated to collect samples. Discrete samples were collected by the automatic sampler at 15-minute intervals during a period of runoff. Near or at the end of the runoff event, sampling was completed and the discrete samples from a site were combined into a single composite sample. At the remaining stream sites, where the installation of automatic samplers was impractical, multiple grab samples were collected from the stream during runoff events by USGS personnel, either by wading when possible or from watercraft.

Eighteen of the 21 wells sampled for this study had permanently-installed pumps from which samples were collected from raw-water spigots at or near the wellhead, prior to any pressure tanks or water treatment. Well LR-67-07-808 (hereinafter, TSU-Artesian well; fig. 2, map identifier W7; table 3) was a flowing well from which a sample was collected at the wellhead. The Fish Hatchery and LR-67-01-826 (hereinafter, TSU-West Campus; fig. 2, map identifier W6; table 3) wells were monitoring wells into which a submersible pump equipped with 3/8-inch Teflon-lined tubing was lowered to collect a raw-water sample. Before samples were collected and processed, all wells were pumped (or allowed to flow in the cases of artesian wells) to remove 3 casing volumes of water, or until field measurements of water temperature, specific conductance, $\mathrm{pH}$, turbidity, and dissolved-oxygen concentration stabilized. This purging of the well was done to ensure that the samples collected were representative of water from the aquifer (U.S. Geological Survey, variously dated). Field measurements were considered stable when five consecutive readings collected every 5 minutes were within the stabilization criteria for the individual property (Wilde and Radtke, 1998).

Spring orifices were sampled using one of two methods. Grab samples were collected at spring orifices accessible by wading and placing the closed collection container directly into the orifice and then opening, filling, and closing the collection container while still underwater in the spring orifice. A stainless steel intake attached to 3/8-inch Teflon-lined tubing was inserted into orifices to collect samples from springs that were not accessible by wading. A peristaltic pump then was used to draw water through the tubing to the surface for the recording of field measurements and sample collection.

\section{Sample Analysis}

Four laboratories analyzed samples for this study. The USGS National Water Quality Laboratory (NWQL) in Lakewood, Colo., was the primary laboratory and analyzed samples for TDS, major ions (calcium, magnesium, potassium, sodium, bromide, chloride, fluoride, silica, and sulfate), nutrients (ammonia, nitrite, nitrite plus nitrate, total nitrogen, orthophosphate, and phosphorus), trace elements (aluminum, antimony, arsenic, barium, beryllium, boron, cadmium, chromium, cobalt, copper, iron, lead, lithium, manganese, molybdenum, nickel, selenium, silver, strontium, thallium, uranium, vanadium, and zinc), DOC, and TOC concentrations. The National Ocean Sciences Accelerator Mass Spectrometry Facility at the Woods Hole Oceanographic Institution in Woods Hole, Mass., was contracted by the NWQL to analyze samples for carbon- $13\left({ }^{13} \mathrm{C}\right)$ to carbon- $12\left({ }^{12} \mathrm{C}\right)$ isotopic ratios; the USGS Reston Stable Isotope Laboratory (RSIL) in Reston, Va., analyzed samples for oxygen-18 $\left({ }^{18} \mathrm{O}\right)$ to oxygen-16 $\left({ }^{16} \mathrm{O}\right)$ and deuterium $\left({ }^{2} \mathrm{H}\right)$ to protium $\left({ }^{1} \mathrm{H}\right)$ isotopic ratios; and the USGS National Research Program Laboratory in Menlo Park, Calif., analyzed samples for strontium- $87\left({ }^{87} \mathrm{Sr}\right)$ to strontium- $86\left({ }^{86} \mathrm{Sr}\right)$ isotopic ratios.

Anion and major cation concentrations were measured using ion-exchange chromatography and inductively coupled plasma-atomic emissions spectrometry (ICP-AES), respectively, as described by Fishman (1993). Nutrient concentrations were measured using approved methods as described by Fishman (1993) and Patton and Kryskalla (2003). Trace element concentrations were measured using collision-reaction cell inductively coupled plasma-mass spectrometry (cICP-MS) (Garbarino and others, 2006) or inductively coupled plasmamass spectrometry (ICP-MS) (Faires, 1993; Garbarino, 1999). DOC concentrations were measured using ultravioletpromoted persulfated oxidation and infrared spectrometry as described by Brenton and Arnett (1993). TOC concentrations were measured using high-temperature combustion method 5310B as described by Clesceri and others (1998). ${ }^{13} \mathrm{C}$ and ${ }^{12} \mathrm{C}$ isotope compositions were measured using techniques described by Vogel and others $(1985) .{ }^{18} \mathrm{O},{ }^{16} \mathrm{O},{ }^{2} \mathrm{H}$, and ${ }^{1} \mathrm{H}$ isotope compositions were measured using techniques described in Révész and Coplen (2008a and 2008b). ${ }^{87} \mathrm{Sr}$ and ${ }^{86} \mathrm{Sr}$ isotope compositions were measured using techniques described by Bayless and others (2004).

\section{Results Reporting}

The analytical quantification procedure used by the NWQL for reporting results is based on long-term method detection levels (LT-MDL) and laboratory reporting levels (LRL). The LT-MDL concentrations are defined for most 
analytical methods at the NWQL to limit the false positive rate to less than or equal to 1 percent. The LRL is defined as twice the LT-MDL to limit the occurrence of false negative detections to less than or equal to 1 percent (Childress and others, 1999). A constituent concentration greater than the LT-MDL and less than the LRL indicates the detection of a constituent is considered likely, but quantification is considered questionable. These results are considered estimated and the remark code of "E" (estimated) is assigned by the laboratory for these results (Childress and others, 1999). A constituent concentration less than the LT-MDL is considered a nondetection and is reported as less than the LRL.

The LT-MDLs and LRLs changed for some constituents during the study because the NWQL reassesses their method detection and reporting level values on a regular basis. Table 5 shows all the LT-MDLs and LRLs that were in effect during the study for the constituents sampled. The table also shows the range of dates for the samples affected by the various LT-MDLs and LRLs by constituent.

Stable environmental isotopes of carbon, oxygen, and hydrogen are measured as the ratio of the two most abundant isotopes of a given element. The most abundant stable isotopes of carbon are ${ }^{13} \mathrm{C}$ and ${ }^{12} \mathrm{C}$, the most abundant isotopes of oxygen are ${ }^{18} \mathrm{O}$ and ${ }^{16} \mathrm{O}$, and the most abundant isotopes of hydrogen are ${ }^{2} \mathrm{H}$ and ${ }^{1} \mathrm{H}$ (Clark and Fritz, 1997). Stable isotopic compositions of oxygen, hydrogen, and carbon are reported as relative isotope ratios in parts per thousand (per mil) using the standard delta notation (Coplen and others, 2002):

$$
\delta^{\mathrm{i} X}=\left[\mathrm{R}_{\text {sample }} / \mathrm{R}_{\text {standard }}-1\right] \times 1,000
$$

where

$$
\begin{gathered}
\delta^{\mathrm{i} X} \quad \begin{array}{l}
\text { is the heavier isotope }\left({ }^{13} \mathrm{C},{ }^{18} \mathrm{O}, \text { or }{ }^{2} \mathrm{H}\right), \\
\mathrm{R}_{\text {sample }} \\
\text { is the ratio of the abundance of the heavier } \\
\text { isotope to the lighter isotope }\left({ }^{12} \mathrm{C},{ }^{16} \mathrm{O}\right. \text {, or } \\
\left.{ }^{1} \mathrm{H}\right) \text { in the sample, and }
\end{array} \\
\mathrm{R}_{\text {standard }} \\
\text { is the ratio of the abundance of the heavier } \\
\text { isotope to the lighter isotope in the } \\
\text { standard material. }
\end{gathered}
$$

The standard material for carbon is Vienna PeeDee Belemnite (VPDB), which is assigned a $\delta^{13} \mathrm{C}$ value of 0 per mil (Fritz and Fontes, 1980). The standard material for oxygen and hydrogen is Vienna Standard Mean Ocean Water (VSMOW), which is assigned $\delta^{18} \mathrm{O}$ and $\delta^{2} \mathrm{H}$ values of 0 per mil $\left(\delta^{2} \mathrm{H}\right.$ can also be written as $\delta \mathrm{D}$ because the common name of the heavier isotope of hydrogen, hydrogen-2, is deuterium) (Fritz and Fontes, 1980). Positive values indicate enrichment of the heavier isotope and negative values indicate depletion of the heavier isotope, compared to the ratios observed in the standard material.

The stable isotopes ${ }^{86} \mathrm{Sr}$ and ${ }^{87} \mathrm{Sr}$, while not the most abundant isotopes of strontium, can be useful in determining flow paths in hydrogeologic investigations (Clark and Fritz, 1997). Stable strontium isotopic compositions are reported as dimensionless molar ratios. The molar ratio ${ }^{87 / 86} \mathrm{Sr}$ is computed by dividing the concentration of ${ }^{87} \mathrm{Sr}$, in moles per liter, by the concentration of ${ }^{86} \mathrm{Sr}$, in moles per liter.

\section{Quality Control}

Quality-assurance procedures outlined in the "National Field Manual for the Collection of Water-Quality Data" (U.S. Geological Survey, variously dated) were followed for collecting and processing water-quality samples. Quality-assurance samples collected for this study included blank and replicate samples.

Equipment and field blank samples were processed beginning in November 2008 and continued throughout the study period to evaluate the extent of contamination introduced during sampling, sample processing, shipping, or laboratory analysis. Blank samples were processed using water certified to contain undetectable concentrations of constituents to be analyzed. The water used for the inorganic constituent samples was distilled, deionized water. The water used for the DOC and TOC samples was pesticide-grade blank water. Both types of blank water were obtained from the NWQL. A total of 293 concentrations for 42 constituents were analyzed in 13 blank samples (appendix 2). Detectable concentrations were measured in 25 of the 293 values measured in the blank samples. Twelve of the 25 detected concentrations were estimated concentrations. Of the 13 remaining detected concentrations, cobalt and manganese each were detected in four samples; chloride was detected in two samples; and silica, molybdenum, and zinc each were detected in one sample. Four cobalt concentrations; three manganese and DOC concentrations; and one concentration each of fluoride, molybdenum, antimony, zinc, and TOC in environmental samples were rejected and not reported because the associated blank sample had a concentration greater than that of the environmental sample.

Eighteen replicate samples (appendix 1) were collected from 14 sites to evaluate potential bias, variability, or contamination introduced during sample collection, processing, or laboratory analysis. Replicate samples were compared to the associated environmental samples by calculating the relative percent difference (RPD) for each pair of detected constituents (appendix 1). The RPD was not computed for a constituent pair if one or both of the concentrations were nondetections or were reported as estimated. Of the total 775 constituent pairs, RPD was computed for 577 pairs. RPD was computed using the equation

$$
\mathrm{RPD}=\left|\mathrm{C}_{1}-\mathrm{C}_{2}\right| /\left(\left(\mathrm{C}_{1}+\mathrm{C}_{2}\right) / 2\right) \times 100,
$$

where

$$
\begin{aligned}
& \mathrm{C}_{1} \quad \text { is the concentration from environmental } \\
& \text { sample; and } \\
& \mathrm{C}_{2} \quad \text { is the concentration from replicate sample. }
\end{aligned}
$$

RPDs of 15 percent or less were judged to indicate good agreement between analytical results if the concentrations are sufficiently large compared to the LRL. For 577 sample pairs 
Table 5. Summary of long-term method detection levels and laboratory reporting levels for constituents sampled for San Marcos Springs recharge study, Bexar, Comal, and Hays Counties, Texas, November 2008-December 2010.

[LT-MDL, long-term method detection level; LRL, laboratory reporting level; mg/L, milligrams per liter; N/A, not applicable; $\mu \mathrm{g} / \mathrm{L}, \mathrm{micrograms}$ per liter]

\begin{tabular}{|c|c|c|c|c|c|c|c|c|c|}
\hline Constituent & $\begin{array}{c}\text { First } \\
\text { sample } \\
\text { date }\end{array}$ & $\begin{array}{c}\text { Last } \\
\text { sample } \\
\text { date }\end{array}$ & $\begin{array}{l}\text { LT- } \\
\text { MDL }\end{array}$ & LRL & Constituent & $\begin{array}{c}\text { First } \\
\text { sample } \\
\text { date }\end{array}$ & $\begin{array}{c}\text { Last } \\
\text { sample } \\
\text { date }\end{array}$ & $\begin{array}{l}\text { LT- } \\
\text { MDL }\end{array}$ & LRL \\
\hline \multirow{2}{*}{$\begin{array}{l}\text { Total dissolved } \\
\text { solids (mg/L) }\end{array}$} & $11 / 25 / 2008$ & $12 / 3 / 2010$ & N/A & 10 & \multirow[t]{3}{*}{ Cobalt $(\mu \mathrm{g} / \mathrm{L})$} & $11 / 25 / 2008$ & $9 / 24 / 2009$ & 0.01 & 0.02 \\
\hline & & & & & & 10/3/2009 & 9/30/2010 & .005 & .010 \\
\hline \multirow[t]{2}{*}{ Calcium (mg/L) } & $11 / 25 / 2008$ & $9 / 24 / 2009$ & .01 & .02 & & $10 / 1 / 2010$ & $12 / 3 / 2010$ & .02 & .04 \\
\hline & $10 / 3 / 2009$ & $12 / 3 / 2010$ & .022 & .044 & Copper $(\mu \mathrm{g} / \mathrm{L})$ & $11 / 25 / 2008$ & $12 / 3 / 2010$ & .5 & 1 \\
\hline \multirow{2}{*}{$\begin{array}{l}\text { Magnesium } \\
\quad(\mathrm{mg} / \mathrm{L})\end{array}$} & $11 / 25 / 2008$ & $9 / 24 / 2009$ & .006 & .012 & \multirow[t]{3}{*}{ Iron $(\mu \mathrm{g} / \mathrm{L})$} & $11 / 25 / 2008$ & $9 / 24 / 2009$ & 2 & 4 \\
\hline & $10 / 3 / 2009$ & $12 / 3 / 2010$ & .008 & .016 & & $10 / 3 / 2009$ & $9 / 30 / 2010$ & 3 & 6 \\
\hline \multirow[t]{3}{*}{ Potassium $(\mathrm{mg} / \mathrm{L})$} & $11 / 25 / 2008$ & $9 / 24 / 2009$ & .03 & .06 & & $10 / 1 / 2010$ & $12 / 3 / 2010$ & 3.2 & 6.4 \\
\hline & $10 / 3 / 2009$ & $9 / 30 / 2010$ & .032 & .064 & \multirow[t]{3}{*}{ Lead $(\mu \mathrm{g} / \mathrm{L})$} & $11 / 25 / 2008$ & $9 / 24 / 2009$ & .03 & .06 \\
\hline & $10 / 1 / 2010$ & $12 / 3 / 2010$ & .022 & .044 & & $10 / 3 / 2009$ & $9 / 30 / 2010$ & .015 & .030 \\
\hline \multirow[t]{3}{*}{ Sodium (mg/L) } & $11 / 25 / 2008$ & $9 / 24 / 2009$ & .06 & .12 & & $10 / 1 / 2010$ & $12 / 3 / 2010$ & .015 & .03 \\
\hline & $10 / 3 / 2009$ & 9/30/2010 & .05 & .1 & \multirow[t]{2}{*}{ Lithium $(\mu \mathrm{g} / \mathrm{L})$} & $11 / 25 / 2008$ & $9 / 24 / 2009$ & .5 & 1 \\
\hline & $10 / 1 / 2010$ & $12 / 3 / 2010$ & .06 & .12 & & $10 / 3 / 2009$ & $12 / 3 / 2010$ & .22 & .44 \\
\hline \multirow[t]{2}{*}{ Bromide $(\mathrm{mg} / \mathrm{L})$} & $11 / 25 / 2008$ & 9/30/2010 & .01 & .02 & \multirow[t]{2}{*}{ Manganese $(\mu \mathrm{g} / \mathrm{L})$} & $11 / 25 / 2008$ & $9 / 24 / 2009$ & .1 & .2 \\
\hline & $10 / 1 / 2010$ & $12 / 3 / 2010$ & .010 & .020 & & $10 / 3 / 2009$ & $12 / 3 / 2010$ & .13 & .26 \\
\hline Chloride (mg/L) & $11 / 25 / 2008$ & $12 / 3 / 2010$ & .06 & .12 & \multirow{2}{*}{$\begin{array}{l}\text { Molybdenum } \\
(\mu \mathrm{g} / \mathrm{L})\end{array}$} & $11 / 25 / 2008$ & $9 / 24 / 2009$ & .01 & .02 \\
\hline Fluoride (mg/L) & $11 / 25 / 2008$ & $12 / 3 / 2010$ & .04 & .08 & & $10 / 3 / 2009$ & $12 / 3 / 2010$ & .014 & .028 \\
\hline \multirow[t]{2}{*}{ Silica (mg/L) } & $11 / 25 / 2008$ & $9 / 24 / 2009$ & .01 & .02 & \multirow[t]{2}{*}{$\operatorname{Nickel}(\mu \mathrm{g} / \mathrm{L})$} & $11 / 25 / 2008$ & $9 / 30 / 2010$ & .06 & .12 \\
\hline & $10 / 3 / 2009$ & $12 / 3 / 2010$ & .029 & .058 & & $10 / 1 / 2010$ & $12 / 3 / 2010$ & .09 & .18 \\
\hline Sulfate (mg/L) & $11 / 25 / 2008$ & $12 / 3 / 2010$ & .09 & .18 & \multirow[t]{3}{*}{ Silver $(\mu \mathrm{g} / \mathrm{L})$} & $11 / 25 / 2008$ & $9 / 24 / 2009$ & .004 & .008 \\
\hline \multirow{3}{*}{$\begin{array}{l}\text { Dissolved am- } \\
\text { monia nitrogen } \\
(\mathrm{mg} / \mathrm{L})\end{array}$} & $11 / 25 / 2008$ & 9/30/2010 & .01 & .02 & & $10 / 3 / 2009$ & $9 / 30 / 2010$ & .005 & .010 \\
\hline & $10 / 1 / 2010$ & $12 / 3 / 2010$ & .010 & .020 & & $10 / 1 / 2010$ & $12 / 3 / 2010$ & .005 & .01 \\
\hline & $11 / 25 / 2008$ & $9 / 30 / 2010$ & .02 & .04 & \multirow[t]{3}{*}{ Strontium $(\mu \mathrm{g} / \mathrm{L})$} & $11 / 25 / 2008$ & 9/24/2009 & .4 & .8 \\
\hline \multirow{2}{*}{$\begin{array}{l}\text { Dissolved nitrate } \\
\text { plus nitrite } \\
\text { nitrogen }(\mathrm{mg} / \mathrm{L})\end{array}$} & $10 / 1 / 2010$ & 12/3/2010 & .02 & .040 & & $10 / 3 / 2009$ & $9 / 30 / 2010$ & .20 & .40 \\
\hline & & & & & & $10 / 1 / 2010$ & $12 / 3 / 2010$ & .2 & .4 \\
\hline \multirow{2}{*}{$\begin{array}{l}\text { Dissolved nitrite } \\
\text { nitrogen }(\mathrm{mg} / \mathrm{L})\end{array}$} & $11 / 25 / 2008$ & 9/30/2010 & .001 & .002 & \multirow[t]{2}{*}{ Thallium $(\mu \mathrm{g} / \mathrm{L})$} & $11 / 25 / 2008$ & $9 / 24 / 2009$ & .02 & .04 \\
\hline & $10 / 1 / 2010$ & $12 / 3 / 2010$ & .0010 & .0020 & & $10 / 3 / 2009$ & $12 / 3 / 2010$ & .010 & .020 \\
\hline \multirow{4}{*}{$\begin{array}{l}\text { Dissolved or- } \\
\text { thophosphate } \\
\text { phosphorus } \\
(\mathrm{mg} / \mathrm{L})\end{array}$} & $11 / 25 / 2008$ & $12 / 3 / 2010$ & .004 & .008 & & $11 / 25 / 2008$ & $12 / 3 / 2010$ & .08 & .16 \\
\hline & & & & & $\operatorname{Zinc}(\mu \mathrm{g} / \mathrm{L})$ & $11 / 25 / 2008$ & $9 / 24 / 2009$ & 1 & 2 \\
\hline & & & & & & $10 / 3 / 2009$ & $12 / 3 / 2010$ & 1.4 & 2.8 \\
\hline & & & & & Antimony $(\mu \mathrm{g} / \mathrm{L})$ & $11 / 25 / 2008$ & $9 / 24 / 2009$ & .02 & .04 \\
\hline Total phosphorus & $11 / 25 / 2008$ & $12 / 3 / 2010$ & .004 & .008 & & $10 / 3 / 2009$ & $12 / 3 / 2010$ & .027 & .054 \\
\hline$(\mathrm{mg} / \mathrm{L})$ & & & & & Arsenic $(\mu \mathrm{g} / \mathrm{L})$ & $11 / 25 / 2008$ & $9 / 24 / 2009$ & .03 & .06 \\
\hline Total nitrogen & $11 / 25 / 2008$ & $9 / 24 / 2009$ & .05 & .1 & & $10 / 3 / 2009$ & $12 / 3 / 2010$ & .022 & .044 \\
\hline (nitrate + nitrite & $10 / 3 / 2009$ & $12 / 3 / 2010$ & .05 & .10 & Boron $(\mu \mathrm{g} / \mathrm{L})$ & $11 / 25 / 2008$ & $9 / 24 / 2009$ & 2 & 4 \\
\hline $\begin{array}{l}+ \text { ammonia } \\
+ \text { organic-N) }\end{array}$ & & & & & & $10 / 3 / 2009$ & 9/30/2010 & 1.4 & 2.8 \\
\hline$(\mathrm{mg} / \mathrm{L})$ & & & & & & $10 / 1 / 2010$ & $12 / 3 / 2010$ & 3 & 6 \\
\hline Aluminum $(\mu \mathrm{g} / \mathrm{L})$ & $11 / 25 / 2008$ & $9 / 24 / 2009$ & 2 & 4 & Selenium $(\mu \mathrm{g} / \mathrm{L})$ & $11 / 25 / 2008$ & $9 / 24 / 2009$ & .03 & .06 \\
\hline & $10 / 3 / 2009$ & $12 / 3 / 2010$ & 1.7 & 3.4 & & $10 / 3 / 2009$ & $9 / 30 / 2010$ & .020 & .040 \\
\hline Barium $(\mu \mathrm{g} / \mathrm{L})$ & $11 / 25 / 2008$ & $9 / 24 / 2009$ & .2 & .4 & & $10 / 1 / 2010$ & $12 / 3 / 2010$ & .03 & .06 \\
\hline & $10 / 3 / 2009$ & $12 / 3 / 2010$ & .07 & .14 & Dissolved organic & $11 / 25 / 2008$ & $9 / 24 / 2009$ & .2 & .4 \\
\hline Beryllium $(\mu \mathrm{g} / \mathrm{L})$ & $11 / 25 / 2008$ & $9 / 24 / 2009$ & .01 & .02 & & $10 / 3 / 2009$ & 9/30/2010 & .33 & .66 \\
\hline & $10 / 3 / 2009$ & $12 / 3 / 2010$ & .006 & .012 & & $10 / 1 / 2010$ & $12 / 3 / 2010$ & .15 & .30 \\
\hline Cadmium $(\mu \mathrm{g} / \mathrm{L})$ & $11 / 25 / 2008$ & $9 / 30 / 2010$ & .01 & .02 & $\begin{array}{l}\text { Total organic } \\
\text { carbon }(\mathrm{mg} / \mathrm{L})\end{array}$ & $11 / 25 / 2008$ & $12 / 3 / 2010$ & .3 & .6 \\
\hline & $10 / 1 / 2010$ & $12 / 3 / 2010$ & .016 & .032 & $\begin{array}{l}\text { carbon }(\mathrm{mg} / \mathrm{L}) \\
\text { ranium }(\mu \mathrm{g} / \mathrm{L})\end{array}$ & $11 / 25 / 2008$ & 9/24/2009 & & \\
\hline Chromium $(\mu \mathrm{g} / \mathrm{L})$ & $11 / 25 / 2008$ & $12 / 3 / 2010$ & .06 & .12 & Uranium $(\mu \mathrm{g} / \mathrm{L})$ & $10 / 3 / 2009$ & $12 / 3 / 2010$ & .004 & $\begin{array}{l}.000 \\
.008\end{array}$ \\
\hline
\end{tabular}


for which the RPD was computed, the RPD was within 15 percent for 50 of 51 sample pairs for nutrients, 161 of 165 sample pairs of major ions, all 18 sample pairs of TDS, 203 of 241 sample pairs for trace elements, 11 of 15 sample pairs of DOC and TOC, and 69 of 71 sample pairs for isotopes. The non-zero RPDs between environmental and replicate samples collected for nutrients, major ions, TDS, trace elements, DOC, and TOC for this study ranged from 0.20 percent for calcium at spring DX-68-15-901 (hereinafter, Hueco Spring; fig. 2, map identifier S4) to 129 percent for chromium in well DX-68-16707 (hereinafter, 4D; fig. 2, map identifier W1) with a median value of 3.28 percent. The non-zero RPDs between environmental and replicate samples collected for isotopes for this study ranged from 0.001 percent for ${ }^{87 / 86} \mathrm{Sr}$ in seven sample pairs to 23.9 percent for $\delta^{13} \mathrm{C}$ at well DX-68-08-701 (hereinafter, Riedel; fig. 2, map identifier W10) with a median of 0.65 percent. RPDs of zero were computed for 20 sample pairs for nutrients, 28 sample pairs for major ions, 3 sample pairs for TDS, 71 sample pairs for trace elements, 3 sample pairs for DOC and TOC, and 3 sample pairs for isotopes. Thirty-three of the 49 sample pairs with RPDs exceeding 15 percent had concentrations that were not sufficiently large compared to the LRL, resulting in a large RPD from a small difference between concentrations.

\section{Continuous Water-Quality Monitors}

Physicochemical properties were collected at six sites periodically from February 2009 to December 2010. Dissolved oxygen concentration, $\mathrm{pH}$, specific conductance, water temperature, and turbidity were collected at the Blanco at Halifax site from February 2009 to December 2010. Water temperature and specific conductance were collected in the TSU-West Campus and LR-67-01-809 (hereinafter, Tipps; fig. 2, map identifier W12; table 3) wells. The monitor in the TSU-West Campus well was operated from January 2009 to December 2010, and the monitor in the Tipps well was operated from November 2008 to May 2010. pH, specific conductance, water temperature, and turbidity were collected from the Deep Spring (S5), Diversion Spring (S7), and Weissmuller Spring (S6) orifices of the San Marcos Springs complex (fig. 3). The Deep Spring monitor was operated from February 2009 to December 2010, the Diversion Spring monitor was operated from April 2009 to December 2010, and the Weissmuller Spring monitor was operated from June 2010 to December 2010. Each of the sensors on the monitors was calibrated as described in "National Field Manual for the Collection of Water-Quality Data" (U.S. Geological Survey, variously dated); the continuous monitor and record were maintained as outlined in Wagner and others (2006).

The water-quality monitors measured physicochemical properties of the water, which then were recorded by a data-collection platform (DCP) at the site. The data stored in the DCP were transmitted every 4 hours by satellite telemetry to a geostationary operations environmental satellite (GOES) that transmitted the data through a USGS ground station to the USGS National Water Information System (NWIS) database (U.S. Geological Survey, 2012). Physicochemical properties were measured every 15 minutes by the monitors at the Blanco at Halifax site and in the orifices of Deep, Diversion, and Weissmuller Springs in the San Marcos Springs complex. Physicochemical properties were measured hourly by the monitors in the two wells. Because provisional data from DCP-equipped sites were uploaded every 4 hours to the NWIS database, it was possible to evaluate conditions at these sites in a near real-time manner.

\section{Surface-Water Data}

Three stream sites (Sink Creek, Purgatory Creek, and Blanco at Halifax) were equipped with data collection platforms (DCPs) to transmit data from near real-time stations to NWIS (U.S. Geological Survey, 2012). Approved USGS methods (Rantz and others, 1982; Sauer and Turnipseed, 2010) were used to measure stage (gage height) every 15 minutes upstream from where each stream crosses the exposed rocks of the Edwards Group (Rose, 1972) known as the Edwards aquifer recharge zone (fig. 1). Stage-discharge ratings were developed for the Sink Creek and Blanco at Halifax sites using approved USGS methods (Rantz and others, 1982; Turnipseed and Sauer, 2010). Because of insufficient flow, the number of discharge measurements made at the Purgatory Creek site was not adequate for the development of a stage-discharge rating.

\section{Groundwater Data}

Continuous water-level data were collected in the TSUWest Campus and Tipps wells near the San Marcos Springs complex using methods described by Freeman and others (2004). Each well was equipped with a submersible pressure transducer and a DCP for real-time data transmission to the Internet. Depth to water from land surface was measured hourly and transmitted to the Internet every 4 hours.

\section{Geochemical Data}

\section{Periodic Water-Quality Sample Data}

A total of 305 environmental samples and 18 replicate samples were collected during the study period (appendix 1). The samples were analyzed for TDS, major ion, nutrient, trace element, DOC, and TOC concentrations and several stable isotopic ratios. 


\section{Total Dissolved Solids and Major lons}

A summary of constituents detected in the 303 samples collected for TDS and major ion analysis is shown in table 6 . TDS were present in all samples and ranged from 107 to 1,160 milligrams per liter $(\mathrm{mg} / \mathrm{L})$. A total of 303 environmental samples were analyzed for 9 major ion constituents and TDS. Major ions were detected at concentrations greater than or equal to the LRL in all but 6 of the 2,727 measured values and ranged from an estimated value of $0.01 \mathrm{mg} / \mathrm{L}$ bromide to $561 \mathrm{mg} / \mathrm{L}$ sulfate.

Table 6. Summary of constituent detections greater than or equal to the laboratory reporting level in samples collected for total dissolved solids and major-ion analysis for San Marcos Springs recharge study, Bexar, Comal, and Hays Counties, Texas, November 2008-December 2010.

[mg/L, milligrams per liter; E, estimated]

\begin{tabular}{lccccc}
\hline \multicolumn{1}{c}{ Constituent } & $\begin{array}{c}\text { Num- } \\
\text { ber of } \\
\text { sam- } \\
\text { ples }\end{array}$ & $\begin{array}{c}\text { Num- } \\
\text { ber of } \\
\text { detec- } \\
\text { tions }\end{array}$ & $\begin{array}{c}\text { Minimum } \\
\text { concen- } \\
\text { tration } \\
\text { (mg/L) }\end{array}$ & $\begin{array}{c}\text { Maximum } \\
\text { concen- } \\
\text { tration } \\
\text { (mg/L) }\end{array}$ & $\begin{array}{c}\text { Median } \\
\text { concen- } \\
\text { tration } \\
\text { (mg/L) }\end{array}$ \\
\hline $\begin{array}{l}\text { Total dissolved } \\
\quad 30 \text { sods }\end{array}$ & 303 & 303 & 107 & 1,160 & 332 \\
Calcium & 303 & 303 & 18.6 & 205 & 84.5 \\
Magnesium & 303 & 303 & 1.38 & 85.8 & 17.3 \\
Potassium & 303 & 303 & .46 & 10.6 & 1.35 \\
Sodium & 303 & 303 & .89 & 57.1 & 9.27 \\
Bromide & 303 & 299 & $\mathrm{E} 0.01$ & .24 & .09 \\
Chloride & 303 & 303 & 1.32 & 52.8 & 16.2 \\
Fluoride & 303 & 301 & $\mathrm{E} .04$ & 3.02 & .22 \\
Silica & 303 & 303 & 4.79 & 24.6 & 11.5 \\
Sulfate & 303 & 303 & 2.13 & 561 & 25.0 \\
\hline
\end{tabular}

\section{Nutrients}

A summary of constituents detected in the samples collected for nutrient analysis is shown in table 7. A total of 303 environmental samples were collected for five of the six nutrient constituents. The sixth constituent, total phosphorus was analyzed in 230 samples. Total phosphorus, initially analyzed in groundwater samples to determine its usefulness in ascertaining surface-water/groundwater communication, was determined to be ineffective for this purpose, and groundwater samples were not analyzed for total phosphorus after sampling cycle four. Nutrients (nitrogen or phosphorus constituents) were detected at concentrations greater than or equal to the LRL in 1,175 of the 1,745 measured values (approximately 67 percent) and ranged from an estimated value of $0.001 \mathrm{mg} / \mathrm{L}$ dissolved nitrite nitrogen to $3.38 \mathrm{mg} / \mathrm{L}$ dissolved nitrite plus nitrate nitrogen and total nitrogen.

\section{Trace Elements}

A summary of the constituents detected in the samples collected for trace element analysis is shown in table 8. A total of 303 environmental samples were analyzed for 23 trace element constituents. Trace elements were detected at concentrations greater than or equal to the LRL in 4,955 of the 6,969 measured values (approximately 71 percent) and ranged from an estimated value of $0.01 \mu \mathrm{g} / \mathrm{L}$ beryllium, cadmium, silver, and thallium to $43,600 \mu \mathrm{g} / \mathrm{L}$ strontium.

\section{Dissolved Organic Carbon and Total Organic Carbon}

A summary of the constituents detected in the samples collected for the measurement of DOC and TOC is shown in table 9. DOC concentrations were detected at concentrations greater than or equal to the LRL in 273 of 303 measured values (approximately 90 percent) and TOC concentrations were

Table 7. Summary of constituent detections greater than or equal to the laboratory reporting level in samples collected for nutrient analysis for San Marcos Springs recharge study, Bexar, Comal, and Hays Counties, Texas, November 2008-December 2010.

[mg/L, milligrams per liter; E, estimated]

\begin{tabular}{lccccc}
\hline \multicolumn{1}{c}{ Constituent } & $\begin{array}{c}\text { Number of } \\
\text { samples }\end{array}$ & $\begin{array}{c}\text { Number of } \\
\text { detections }\end{array}$ & $\begin{array}{c}\text { Minimum } \\
\text { concentration } \\
\text { (mg/L) }\end{array}$ & $\begin{array}{c}\text { Maximum } \\
\text { concentration } \\
\text { (mg/L) }\end{array}$ & $\begin{array}{c}\text { Median } \\
\text { concentration } \\
\text { (mg/L) }\end{array}$ \\
\hline Dissolved ammonia nitrogen & 303 & 60 & $\mathrm{E} 0.010$ & 0.32 & $\mathrm{E} 0.16$ \\
Dissolved nitrite plus nitrate nitrogen & 303 & 283 & $\mathrm{E} .02$ & 3.38 & 1.13 \\
Dissolved nitrite nitrogen & 303 & 56 & $\mathrm{E} .001$ & .028 & .003 \\
Dissolved orthophosphate phosphorus & 303 & 297 & $\mathrm{E} .004$ & .056 & .01 \\
Total phosphorus & 230 & 188 & $\mathrm{E} .004$ & .227 & $\mathrm{E} .008$ \\
$\begin{array}{l}\text { Total nitrogen (nitrate + nitrite + } \\
\text { ammonia + organic-nitrogen) }\end{array}$ & 303 & 291 & $\mathrm{E} .05$ & 3.38 & 1.17 \\
\hline
\end{tabular}


Table 8. Summary of constituent detections greater than or equal to the laboratory reporting level in samples collected for trace element analysis for San Marcos Springs recharge study, Bexar, Comal, and Hays Counties, Texas, November 2008December 2010.

$[\mu \mathrm{g} / \mathrm{L}$, micrograms per liter; E, estimated]

\begin{tabular}{|c|c|c|c|c|c|}
\hline Constituent & $\begin{array}{l}\text { Num- } \\
\text { ber of } \\
\text { sam- } \\
\text { ples }\end{array}$ & $\begin{array}{c}\text { Num- } \\
\text { ber of } \\
\text { detec- } \\
\text { tions }\end{array}$ & $\begin{array}{c}\text { Minimum } \\
\text { concen- } \\
\text { tration } \\
(\mu \mathrm{g} / \mathrm{L})\end{array}$ & $\begin{array}{c}\text { Maximum } \\
\text { concen- } \\
\text { tration } \\
(\mu \mathrm{g} / \mathrm{L})\end{array}$ & $\begin{array}{c}\text { Median } \\
\text { concen- } \\
\text { tration } \\
(\mu \mathrm{g} / \mathrm{L})\end{array}$ \\
\hline Aluminum & 303 & 66 & E1.8 & 233 & 3.9 \\
\hline Barium & 303 & 302 & 6 & 131 & 36 \\
\hline Beryllium & 303 & 9 & E.01 & .04 & .01 \\
\hline Cadmium & 303 & 122 & E.01 & .96 & E.02 \\
\hline Chromium & 303 & 236 & E.06 & .35 & .13 \\
\hline Cobalt & 303 & 299 & .02 & 2.3 & .16 \\
\hline Copper & 303 & 126 & E. 50 & 8.8 & 1.1 \\
\hline Iron & 303 & 108 & E2 & 179 & 5.5 \\
\hline Lead & 303 & 153 & E.02 & 1.76 & .008 \\
\hline Lithium & 303 & 301 & E. 2 & 69.8 & 4 \\
\hline Manganese & 303 & 256 & E. 1 & 33.1 & .35 \\
\hline Molybdenum & 303 & 302 & .1 & 33.4 & .7 \\
\hline Nickel & 303 & 302 & .16 & 2.8 & .755 \\
\hline Silver & 303 & 39 & E.01 & .11 & .02 \\
\hline Strontium & 303 & 302 & 10.3 & 43,600 & 509.5 \\
\hline Thallium & 303 & 158 & E.01 & .05 & E.02 \\
\hline Vanadium & 303 & 290 & E.10 & 3.5 & 1.9 \\
\hline Zinc & 303 & 138 & E1.1 & 46.9 & 4.15 \\
\hline Antimony & 303 & 252 & E.02 & .18 & .04 \\
\hline Arsenic & 303 & 301 & E.03 & 2.4 & .34 \\
\hline Boron & 303 & 302 & 17 & 669 & 52 \\
\hline Selenium & 303 & 289 & .06 & 1.3 & .44 \\
\hline Uranium & 303 & 302 & .01 & 1.58 & .75 \\
\hline
\end{tabular}

detected at concentrations greater than or equal to the LRL in 260 of 303 measured values (approximately 86 percent). DOC values ranged from an estimated value of $0.2 \mathrm{mg} / \mathrm{L}$ to $14.8 \mathrm{mg} / \mathrm{L}$ and TOC values ranged from an estimated value of $0.3 \mathrm{mg} / \mathrm{L}$ to $34.1 \mathrm{mg} / \mathrm{L}$.

\section{Isotopes}

A summary of the stable isotopic ratios collected for this study is shown in table 10 . The values of the isotopic ratio ${ }^{87 / 86} \mathrm{Sr}$ ranged from 0.70743 to 0.70865 for 303 samples collected from the 37 periodic water-quality sites. The values of the isotopic ratio $\delta^{13} \mathrm{C}$ ranged from -14.38 to -1.18 per mil in 302 samples collected from the 37 periodic water-quality sites. Isotopic ratios $\delta^{18} \mathrm{O}$ and $\delta \mathrm{D}$ were measured in 305 samples
Table 9. Summary of constituent detections greater than or equal to the laboratory reporting level in samples collected for dissolved organic carbon and total organic carbon analysis for San Marcos Springs recharge study, Bexar, Comal, and Hays Counties, Texas, November 2008-December 2010.

[mg/L, milligrams per liter; E, estimated]

\begin{tabular}{cccccc}
\hline Constituent & $\begin{array}{c}\text { Num- } \\
\text { ber of } \\
\text { samples }\end{array}$ & $\begin{array}{c}\text { Number } \\
\text { of de- } \\
\text { tections }\end{array}$ & $\begin{array}{c}\text { Minimum } \\
\text { concen- } \\
\text { tration } \\
\text { (mg/L) }\end{array}$ & $\begin{array}{c}\text { Maximum } \\
\text { concen- } \\
\text { tration } \\
\text { (mg/L) }\end{array}$ & $\begin{array}{c}\text { Median } \\
\text { concen- } \\
\text { tration } \\
\text { (mg/L) }\end{array}$ \\
\hline $\begin{array}{c}\text { Dissolved } \\
\text { organic } \\
\text { carbon }\end{array}$ & 303 & 273 & E0.2 & 14.8 & E0.5 \\
$\begin{array}{c}\text { Total } \\
\text { organic } \\
\text { carbon }\end{array}$ & 303 & 260 & E.3 & 34.1 & .75 \\
\hline
\end{tabular}

collected from the 37 periodic water-quality sites. $\delta^{18} \mathrm{O}$ values ranged from -14.46 to 2.54 per mil and $\delta \mathrm{D}$ values ranged from -103 to 6.1 per mil in the 305 samples. In addition to the periodic water-quality sites, $\delta^{18} \mathrm{O}$ and $\delta \mathrm{D}$ isotopic ratios also were measured in 24 precipitation samples. $\delta^{18} \mathrm{O}$ values ranged from -19.13 to -0.32 per mil and $\delta \mathrm{D}$ values ranged from -139 to 8.9 per mil in the 24 precipitation samples.

\section{Water-Quality Monitor Data}

The data collected using the water-quality monitor at the Blanco at Halifax site are reported in appendixes 3.1-3.5. Daily mean dissolved oxygen concentrations at the site ranged from $6.7 \mathrm{mg} / \mathrm{L}$ on July 8,2010 , and September 1, 2010, to $12.9 \mathrm{mg} / \mathrm{L}$ on January 9,2010 . Daily median $\mathrm{pH}$ ranged from 7.6 during April 4-7, 2010, to 8.8 on October 30 and 31, 2010. Daily mean specific conductance ranged from 258 microsiemens per centimeter $(\mu \mathrm{S} / \mathrm{cm})$ on September 11, 2009, to 515 $\mu \mathrm{S} / \mathrm{cm}$ on December 7, 2009. Daily mean water temperature ranged from 4.5 degrees Celsius $\left({ }^{\circ} \mathrm{C}\right)$ on January 9,2010 , to $32.3^{\circ} \mathrm{C}$ on July 8,2009 . Daily mean turbidity values ranged from 3.7 formazin nephelometric units (FNU) on June 10, 2009 , to an estimated value of 2,980 FNU on September 11, 2009.

The data collected using the water-quality monitors at the TSU-West Campus and Tipps wells are reported in appendixes 4.1 and 4.2, and 5.1 and 5.2, respectively. Daily mean specific conductance at the TSU-West Campus well ranged from 617 $\mu \mathrm{S} / \mathrm{cm}$ on August 31, 2010, to $834 \mu \mathrm{S} / \mathrm{cm}$ on July 20, 2009. Daily mean water temperature remained a constant $22.3^{\circ} \mathrm{C}$ during the entire study. Daily mean specific conductance at the Tipps well ranged from $591 \mu \mathrm{S} / \mathrm{cm}$ on December 13 and 14, 2009 , to $984 \mu \mathrm{S} / \mathrm{cm}$ on September 3, 2009. Daily mean water temperature ranged from $18.7^{\circ} \mathrm{C}$ during January $10-12,2010$, to $21.5^{\circ} \mathrm{C}$ during October $2-13,2009$. 
Table 10. Summary of constituent detections in samples collected for isotope analysis for San Marcos Springs recharge study, Bexar, Comal, and Hays Counties, Texas, November 2008December 2010.

$\left[{ }^{87 / 86} \mathrm{Sr}\right.$, strontium-87/strontium-86; $\delta^{13} \mathrm{C}$, delta carbon-13; per mil, parts per thousand; $\delta \mathrm{D}$, delta deuterium; $\delta^{18} \mathrm{O}$, delta oxygen-18]

\begin{tabular}{lcccc}
\hline Isotopic ratio & $\begin{array}{c}\text { Number of } \\
\text { samples }\end{array}$ & $\begin{array}{c}\text { Minimum } \\
\text { ratio }\end{array}$ & $\begin{array}{c}\text { Maximum } \\
\text { ratio }\end{array}$ & $\begin{array}{c}\text { Median } \\
\text { ratio }\end{array}$ \\
\hline & \multicolumn{4}{c}{ Periodic water-quality samples } \\
\hline${ }^{87 / 86} \mathrm{Sr}$ & 303 & 0.70743 & 0.70865 & 0.70788 \\
$\delta^{13} \mathrm{C}$ (per mil) & 302 & -14.38 & -1.18 & -9.09 \\
$\delta \mathrm{D}$ (per mil) & 305 & -103 & 6.1 & -23.1 \\
$\delta^{18} \mathrm{O}$ (per mil) & 305 & -14.46 & 2.54 & -4.19 \\
\hline \multicolumn{5}{c}{ Precipitation samples } \\
\hline$\delta \mathrm{D}$ (per mil) & 24 & -139 & 8.9 & -21.35 \\
$\delta^{18} \mathrm{O}$ (per mil) & 24 & -19.13 & -.32 & -4.17 \\
\hline
\end{tabular}

The data collected using the water-quality monitor at Deep Spring are reported in appendixes 6.1-6.4. Daily median $\mathrm{pH}$ at the site ranged from 6.9 on May 8-12, 2009, to 7.2 on various days throughout 2009 and 2010. Daily mean specific conductance ranged from $602 \mu \mathrm{S} / \mathrm{cm}$ on December 9,12 , and 13,2010 , to $634 \mu \mathrm{S} / \mathrm{cm}$ on July 8,2009 . Daily mean water temperature ranged from $21.5^{\circ} \mathrm{C}$ on December 4, 2009, and January 8,2010 , to $22.4^{\circ} \mathrm{C}$ for 14 days in May 2009 and on July 4, 2009. Daily mean turbidity values ranged from less than the accuracy of the turbidity probe (less than $0.3 \mathrm{FNU}$ ) during the majority of the period of record to $0.7 \mathrm{FNU}$ on December 26, 2010.

The data collected using the water-quality monitor at Diversion Spring are reported in appendixes 7.1-7.4. Daily median $\mathrm{pH}$ at the site ranged from 7.0 near the beginning and end of the period of record to 7.4 from March 27-June 18, 2010. Daily mean specific conductance ranged from 586 $\mu \mathrm{S} / \mathrm{cm}$ during December $21-31,2010$, to $609 \mu \mathrm{S} / \mathrm{cm}$ during February 14-16 and 21-24, 2010. Daily mean water temperature ranged from $21.6^{\circ} \mathrm{C}$ during February 2010 and December 2010 to $22.2^{\circ} \mathrm{C}$ for 13 days in August and September 2009 . Daily mean turbidity values were less than $0.3 \mathrm{FNU}$ during the entire period of record.

The data collected using the water-quality monitor at Weissmuller Spring are reported in appendixes 8.1-8.4. Daily median $\mathrm{pH}$ at the site ranged from 6.8 on November 9,15 , and 16 and December 4, 2010, to 7.2 during July $2-7,2010$. Daily mean specific conductance ranged from $589 \mu \mathrm{S} / \mathrm{cm}$ during December 7-13, 2010, to $600 \mu \mathrm{S} / \mathrm{cm}$ for 18 days in July 2010. Daily mean water temperature remained a constant $21.5^{\circ} \mathrm{C}$ during the entire study. Daily mean turbidity values ranged from less than 0.3 FNU from June 10-29, 2010, to 0.9 FNU during December 11-13, 2010.

\section{Hydrologic Data}

\section{Surface-Water Data}

The daily mean gage height data collected at the three continuous real-time stream sites (Sink Creek, Purgatory Creek, and Blanco at Halifax) are reported in appendixes 9-11. In addition to gage height data, continuous discharge data were computed using a stage-discharge rating for the Sink Creek and Blanco at Halifax sites and are reported in appendixes 12 and 13. The periodic discharge measurements done at the Sink Creek site and Blanco at Halifax sites from which continuous discharge data were computed are listed in appendix 14 .

\section{Stream Sites}

The daily mean gage height data collected at the Sink Creek, Purgatory Creek, and Blanco at Halifax sites are reported in appendixes 9, 10, and 11, respectively. The Sink Creek and Purgatory Creek sites were dry during most of the study period. During smaller rain events, water pooled upstream from the controls at the two sites resulting in increases in the daily mean gage height, but no flow occurred in the stream channel. The gage height at which flow does not occur in the stream channel is termed the point of zero flow (PZF). The PZF was 1.3 feet above gage datum at the Sink Creek site and was estimated to be 1.0 feet above gage datum at the Purgatory Creek site. The maximum daily mean gage height at the Sink Creek site was 1.91 feet on September 8, 2010. The maximum daily mean gage height at the Purgatory Creek site was 1.70 feet on September 8, 2010. The PZF at the Blanco at Halifax site was not determined because there was always flow at the site. The minimum daily mean gage height was 0.61 feet on July 28 and 29, 2009, and August 10 and 11, 2009. The maximum daily mean gage height was 5.8 feet on September 8, 2010.

The daily mean discharge data computed for the Sink Creek and Blanco at Halifax sites are reported in appendixes 12 and 13, respectively. The minimum daily mean discharge at the Sink Creek site was $0 \mathrm{ft}^{3} / \mathrm{s}$ for a majority of the study period. The maximum daily mean discharge at the site was $25 \mathrm{ft}^{3} / \mathrm{s}$ on September 8, 2010. The minimum daily mean discharge at the Blanco at Halifax site was $2.3 \mathrm{ft}^{3} / \mathrm{s}$ on July 28 and 29, 2009, and August 18 and 19, 2009. The maximum daily mean discharge at the site was estimated to be $3,620 \mathrm{ft}^{3} / \mathrm{s}$ on September 8, 2010.

\section{Periodic Discharge Measurements}

Instantaneous discharge measurements made at the Sink Creek and Blanco at Halifax sites are reported in appendix 14. Two discharge measurements were made at the Sink Creek site on October 4, 2009, and September 8, 2010. Sixteen discharge 
measurements were done at the Blanco at Halifax site from February 2009 to October 2010.

\section{Groundwater Data}

Daily mean depth to water below land surface data collected at the TSU-West Campus and Tipps wells are reported in appendixes 15 and 16, respectively. The daily mean depth to water in the TSU-West Campus well during the study period ranged from 171.76 feet below land surface on February 25, 2010, to 176.64 feet below land surface on September 8, 2009. The daily mean depth to water in the Tipps well during the study period ranged from 22.05 feet below land surface on February 21, 2010, to 28.47 feet below land surface on September 8 and 9, 2009.

\section{Summary}

During 2008-10, the U.S. Geological Survey (USGS), in cooperation with the San Antonio Water System, collected geochemical and hydrologic data in a study area that includes parts of Bexar, Comal, and Hays Counties, Texas, to define and characterize the sources of recharge to San Marcos Springs. Water-quality samples were collected at 9 stream sites, 21 wells, and 7 springs in the study area from November 2008-December 2010. In addition, precipitation samples were collected at one site for analysis of stable isotopes. Continuous water-quality monitors were operated and maintained at one surface-water site, two wells, and three spring sites. Three stream sites were installed for the collection of hydrologic data for this study. Continuous gage height was recorded at all three stream sites, and a stage-discharge rating was developed at two of the sites. Continuous depth to water below land surface also was measured in two wells.

A total of 305 environmental water-quality and 32 quality-assurance samples were collected in 20 sampling cycles (17 routine and 3 storm sampling cycles) during the study period. The samples were analyzed for total dissolved solids (TDS), major ions, nutrients, trace elements, dissolved organic carbon (DOC), and total organic carbon (TOC) concentrations. TDS were present in all 303 samples and ranged from 107 to 1,160 milligrams per liter $(\mathrm{mg} / \mathrm{L})$. Major ions were detected at levels greater than and equal to the laboratory reporting level (LRL)in all but 6 of the 2,727 measured values and ranged from an estimated value of $0.01 \mathrm{mg} / \mathrm{L}$ bromide to $561 \mathrm{mg} / \mathrm{L}$ sulfate. Nutrients were detected at levels greater than and equal to the LRL in 1,175 (approximately 67 percent) of the 1,745 measured values and ranged from estimated value of $0.001 \mathrm{mg} / \mathrm{L}$ dissolved nitrite nitrogen to $3.38 \mathrm{mg} / \mathrm{L}$ dissolved nitrite plus nitrate nitrogen. Trace elements were detected at levels greater than and equal to the LRL in 4,955 (approximately 71 percent) of the 6,969 measured values and ranged from estimated value of 0.01 micrograms per liter $(\mu \mathrm{g} / \mathrm{L})$ in four constituents to $43,600 \mu \mathrm{g} / \mathrm{L}$ strontium.
DOC concentrations were detected at levels greater than and equal to the LRL in 273 (or approximately 90 percent) and TOC concentrations were detected at levels greater than and equal to the LRL in 260 (or approximately 86 percent) of the 303 samples. DOC values ranged from estimated values of 0.2 to $14.8 \mathrm{mg} / \mathrm{L}$ and TOC values ranged from estimated values of 0.3 to $34.1 \mathrm{mg} / \mathrm{L}$.

Values of the isotopic ratio delta carbon-13 $\left(\delta^{13} \mathrm{C}\right)$ ranged from -14.38 to -1.18 parts per thousand (per mil) in 302 samples collected from the 37 periodic water-quality sites. Delta oxygen-18 $\left(\delta^{18} \mathrm{O}\right)$ and delta deuterium $(\delta \mathrm{D})$ isotopic ratios were measured in 305 samples collected from the 37 periodic water-quality sites. $\delta^{18} \mathrm{O}$ values ranged from -14.46 to 2.54 per mil and $\delta \mathrm{D}$ values ranged from -103 to 6.1 per mil in the 305 samples. In addition to the periodic water-quality sites, $\delta^{18} \mathrm{O}$ and $\delta \mathrm{D}$ isotopic ratios also were measured in 24 precipitation samples. $\delta^{18} \mathrm{O}$ values ranged from -19.13 to -0.32 per mil and $\delta \mathrm{D}$ values ranged from -139 to 8.9 per mil in the 24 precipitation samples. The values of strontium-87/ strontium-86 $\left({ }^{87 / 86} \mathrm{Sr}\right)$ isotopic ratios ranged from 0.70743 to 0.70865 in 303 samples collected from the 37 periodic waterquality sites.

Continuous water-quality monitors were used to record physicochemical properties at one surface-water site, two wells, and three spring sites. Dissolved oxygen concentration, $\mathrm{pH}$, specific conductance, water temperature, and turbidity were collected at the Blanco at Halifax site. Specific conductance and water temperature were collected in the TSU-West Campus and Tipps wells. $\mathrm{pH}$, specific conductance, water temperature, and turbidity were collected from the Deep, Diversion, and Weissmuller spring orifices of the San Marcos Springs complex.

Three stream sites equipped with data collection platforms (DCPs) to transmit data to the Internet (real-time stream sites) were installed on two ephemeral streams (Sink Creek and Purgatory Creek) and one perennial stream (Blanco River) for the collection of hydrologic data for this study. Stage-discharge ratings were developed for the Sink Creek and Blanco at Halifax stream sites. The Sink Creek and Purgatory Creek sites were dry during most of the study period. The maximum daily mean gage height at the Sink Creek site was 1.91 feet, which was associated with the maximum daily mean discharge of 25 cubic feet per second $\left(\mathrm{ft}^{3} / \mathrm{s}\right)$. The maximum daily mean gage height at the Purgatory Creek site was 1.70 feet. The minimum daily mean gage height at the Blanco at Halifax site was 0.61 feet, and the maximum daily mean gage height was 5.8 feet. The minimum daily mean discharge at the Blanco at Halifax site was $2.3 \mathrm{ft}^{3} / \mathrm{s}$, and the maximum daily mean discharge at the site was estimated to be $3,620 \mathrm{ft}^{3} / \mathrm{s}$.

Continuous depth to water below land surface data were collected from two wells near the San Marcos Spring complex. The minimum daily mean depth to water below land surface at the TSU-West Campus well during the study period was 171.76 feet, and the maximum daily depth to water below land surface during the study period was 176.64 feet. The minimum daily mean depth to water below land surface at the Tipps 
well during the study period was 22.05 feet, and the maximum daily depth to water below land surface during the study period was 28.47 feet.

\section{References Cited}

Ashworth, J.B., and Hopkins, Janie, 1995, Aquifers of Texas: Texas Water Development Board Report 345, 69 p.

Barker, R.A., and Ardis, A.F., 1996, Hydrogeologic framework of the Edwards-Trinity aquifer system, west-central Texas, in Regional Aquifer-System Analysis-Edwards-Trinity aquifer system: U.S. Geological Survey Professional Paper 1421-B, 61 p., 8 pls.

Bayless, E.R., Bullen, T.D., and Fitzpatrick, J.A., 2004, Use of ${ }^{87} \mathrm{Sr} /{ }^{86} \mathrm{Sr}$ and $\delta^{11} \mathrm{~B}$ to identify slag-affected sediment in southern Lake Michigan: Environmental Science and Technology, v. 38, no. 5, p. 1,330-1,337.

Brenton, R.W., and Arnett, T.L., 1993, Methods of analysis by the U.S. Geological Survey National Water Quality Laboratory-Determination of dissolved organic carbon by UVpromoted persulfate oxidation and infrared spectrometry: U.S. Geological Survey Open-File Report 92-480, 12 p.

Brune, Gunnar, 1975, Major and historical springs of Texas: Texas Water Development Board Report 189, 95 p.

Childress, C.J.O., Foreman, W.T., Connor, B.F., and Maloney, T.J., 1999, New reporting procedures based on longterm method detection levels and some considerations for interpretations of water-quality data provided by the U.S. Geological Survey National Water Quality Laboratory: U.S. Geological Survey Open-File Report 99-193, 19 p.

Clark, I.D., and Fritz, Peter, 1997, Environmental isotopes in hydrogeology: New York, Lewis Publishing, 328 p.

Clesceri, L.S., Greenberg, A.E., and Eaton, A.D., eds., 1998, Standard methods for the examination of water and waste water: Washington, D.C., American Public Health Association [variously paginated].

Coplen, T.B., Hopple, J.A., Böhlke, J.K., Peiser, H.S., Rieder, S.E., Krouse, H.R., Rosman, K.J.R., Ding, T., Vocke, R.D., Jr., Révész, K.M., Lamberty, A., Taylor, P.D.P., and De Bièvre, P., 2002, Compilation of minimum and maximum isotope ratios of selected elements in naturally occurring terrestrial materials and reagents: U.S. Geological Survey Water-Resources Investigations Report 01-4222, 98 p.

Edwards, T.K., and Glysson, D.G., 1999, Field methods for measurement of fluvial sediment: U.S. Geological Survey Techniques of Water-Resources Investigations, book 3, chap. C2, 89 p.
Faires, L.M., 1993, Methods of analysis by the U.S. Geological Survey National Water Quality Laboratory-Determination of metals in water by inductively coupled plasma-mass spectrometry: U.S. Geological Survey Open-File Report 92-634, 28 p.

Fishman, M.J., 1993, Methods of analysis by the U.S. Geological Survey National Water Quality Laboratory-Determination of inorganic and organic constituents in water and fluvial sediments: U.S. Geological Survey Open-File Report 93-125, $217 \mathrm{p}$.

Freeman, L.A., Carpenter, M.C., Rosenberry, D.O., Rousseau, J.P., Unger, Randy, and McLean, J.S., 2004, Use of submersible pressure transducers in water-resources investigations: U.S. Geological Survey Techniques of WaterResources Investigations, book 8, chap. A3, $50 \mathrm{p}$.

Fritz, Peter, and Fontes, J.C., eds., 1980, Handbook of environmental isotope geochemistry, v. 1-The terrestrial environment: Amsterdam, Elsevier, $545 \mathrm{p}$.

Garbarino, J.R., 1999, Methods of analysis by the U.S. Geological Survey National Water Quality LaboratoryDetermination of dissolved arsenic, boron, lithium, selenium, strontium, thallium, and vanadium using inductively coupled plasma-mass spectrometry: U.S. Geological Survey Open-File Report 99-093, 31 p.

Garbarino, J.R., Kanagy, L.K., and Cree, M.E., 2006, Determination of elements in natural water, biota, sediment and soil samples using collision/reaction cell inductively coupled plasma-mass spectrometry, Method ID: I-2020-05: U.S. Geological Survey Techniques and Methods, book 5, chap. B1, 88 p.

Gary, M.O., Gary, R.H., and Asquith, W.H., 2008, Evaluation of acoustic Doppler velocity meters to quantify flow from Comal Springs and San Marcos Springs, Texas: U.S. Geological Survey Scientific Investigations Report 2008-5083, $37 \mathrm{p}$.

Guyton, W.F., and associates, 1979, Geohydrology of Comal, San Marcos, and Hueco Springs, Texas: Department of Water Resources Report 234, 85 p.

Hanson, J.A., and Small, T.A., 1995, Geologic framework and hydrogeologic characteristics of the Edwards aquifer outcrop, Hays County, Texas: U.S. Geological Survey WaterResources Investigations Report 95-4265, 10 p.

Johnson, S.B., and Schindel, G.M., 2008, Evaluation of the option to designate a separate San Marcos pool for critical period management: Edwards Aquifer Authority, San Antonio, Texas, Report 08-01, 109 p.

LBG-Guyton Associates, 2004, Evaluation of augmentation methodologies in support of in-situ refugia at Comal and San Marcos Springs, Texas: Report prepared for Edwards Aquifer Authority, $181 \mathrm{p}$. 
Maclay, R.W., 1995, Geology and hydrology of the Edwards aquifer in the San Antonio area, Texas: U.S. Geological Survey Water-Resources Investigations Report 95-4186, 64 p.

Maclay, R.W., and Land, L.F., 1988, Simulations of flow in the Edwards aquifer, San Antonio region, Texas, and refinement of storage and flow concepts: U.S. Geological Survey Water-Supply Paper 2336-A, 48 p.

Maclay, R.W., and Small, T.A., 1984, Carbonate geology and hydrology of the Edwards aquifer, San Antonio area, Texas, and preliminary interpretations of borehole geophysical and laboratory data on carbonate rocks: U.S. Geological Survey Open-File Report 76-627, 65 p.

Ogden, A.E., Quick, R.A., Rothermel, S.R., and Lundsford, D.L., 1986, Hydrological and hydrochemical investigation of the Edwards aquifer in the San Marcos area, Hays County, Texas: San Marcos, Texas, Edwards Aquifer Research and Data Center, $364 \mathrm{p}$.

Patton, C.J., and Kryskalla, J.R., 2003, Methods of analysis by the U.S. Geological National Water Quality Laboratory: Evaluation of alkaline persulfate digestion as an alternative to Kjeldahl digestion for determination of total and dissolved nitrogen and phosphorus in water: U.S. Geological Survey Water-Resources Investigation Report 2003-4174, $33 \mathrm{p}$.

Puente, Celso, 1976, Statistical analysis of water-level, springflow, and streamflow data for the Edwards aquifer in south-central Texas: U.S. Geological Survey Open-File Report 76-393, 58 p.

Rantz, S.E., and others, 1982, Measurement and computation of streamflow: U.S. Geological Survey Water-Supply Paper 2175 , v. 2, 631 p.

Révész, Kinga, and Coplen, T.B., 2008a, Determination of the $\delta(2 \mathrm{H} / 1 \mathrm{H})$ of water: RSIL lab code 1574 , in chap. C1 of Révész, Kinga, and Coplen, T.B., eds., Methods of the Reston Stable Isotope Laboratory: U.S. Geological Survey Techniques and Methods 10-C1, 27 p., accessed January 18, 2011, at http://pubs.usgs.gov/tm/2007/tm10c1/.

Révész, Kinga, and Coplen, T.B., 2008b, Determination of the $\delta(18 \mathrm{O} / 16 \mathrm{O})$ of water: RSIL lab code 489 , in chap. C2 of Révész, Kinga, and Coplen, T.B., eds., Methods of the Reston Stable Isotope Laboratory: U.S. Geological Survey Techniques and Methods 10-C2, 28 p., accessed January 18, 2011, at http://pubs.usgs.gov/tm/2007/tm10c1/.

Rose, P.R., 1972, Edwards Group, surface and subsurface, central Texas: University of Texas at Austin, Bureau of Economic Geology Report of Investigations, 74 p.

Sauer, V.B., and Turnipseed, D.P., 2010, Stage measurement at gaging stations: U.S. Geological Survey Techniques and Methods book 3, chap. A7, 45 p. (Also available at http://pubs.usgs.gov/tm/tm3-a $7 /$.)

Texas Legislature, 2007, Senate Bill 3, accessed March 18, 2011, at http://www.capitol.state.tx.us/tlodocs/80R/billtext/ pdf/SB00003F.pdf\#navpanes $=0$.

Turnipseed, D.P., and Sauer, V.B., 2010, Discharge measurements at gaging stations: U.S. Geological Survey Techniques and Methods book 3, chap. A8, 87 p. (Also available at http://pubs.usgs.gov/tm/tm3-a8/.)

U.S. Department of Agriculture, 2010, National agriculture imagery program (NAIP): U.S. Department of Agriculture, Farm Service Agency-Aerial photography field office, accessed February 17, 2012, at http://www.fsa.usda.gov/ Internet/FSA_File/naip03_09covermaps.pdf.

U.S. Geological Survey, variously dated, National field manual for the collection of water-quality data: U.S. Geological Survey Techniques of Water-Resources Investigations, book 9, chaps. A1-A9, accessed October 10, 2008, at http://pubs.water.usgs.gov/twri9A.

U.S. Geological Survey, 2010, Annual water data reports: U.S. Geological Survey, accessed on April 5, 2011, at http://wdr.water.usgs.gov.

U.S. Geological Survey, 2012, National Water Information System: U.S. Geological Survey, accessed March 16, 2011, at http://waterdata.usgs.gov/tx/nwis/.

Vogel, J.S., Southon, J.R., Nelson, D.E., and Brown, T.A., 1984, Performance of catalytically condensed carbon for use in accelerator mass spectromentry: Proceedings of the 3rd International Symposium on Accelerator Mass Spectrometry, p. 289-293.

Wagner, R.J., Boulger, R.W., Jr., Oblinger, C.J., and Smith, B.A., 2006, Guidelines and standard procedures for continuous water-quality monitors - Station operation, record computation, and data reporting: U.S. Geological Survey Techniques and Methods 1-D3, $51 \mathrm{p}$. +8 attachments.

Wermund, E.G., 1996, Physiographic regions of Texas: Bureau of Economic Geology, University of Texas at Austin, accessed February 23, 2012, at http://www.beg. utexas.edu/UTopia/images/pagesizemaps/physiography.pdf.

Wilde, F.D., and Radtke, D.B., 1998, National field manual for the collection of water-quality data, field measurements: U.S. Geological Survey Techniques of Water-Resources Investigations, book 9, chap. A6, variable pagination.

William F. Guyton and Associates, 1979, Geohydrology of Comal, San Marcos, and Hueco Springs: Texas Department of Water Resources Report 234, 85 p. 
Publishing support provided by Lafayette Publishing Service Center 



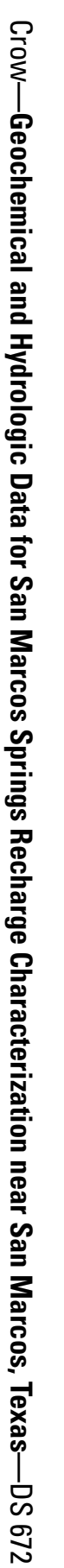

8 Printed on recycled paper 